\title{
Symmetry analysis and equivalence transformations for the construction and reduction of constitutive models
}

\author{
J. F. Ganghoffer ${ }^{1 *}$, R. Rahouadj ${ }^{1}$ and A. Cheviakov ${ }^{2}$
}

\section{${ }^{*}$ Correspondence:}

jean-francois.ganghoffer@univlorraine.fr

'LEM3, UMR CNRS 7239,

Université de Lorraine, 7, rue Félix Savart, 57073 Metz, France Full list of author information is available at the end of the article

\begin{abstract}
A methodology based on Lie analysis is proposed to investigate the mechanical behavior of materials exhibiting experimental master curves. It is based on the idea that the mechanical response of materials is associated with hidden symmetries reflected in the form of the energy functional and the dissipation potential leading to constitutive laws written in the framework of the thermodynamics of irreversible processes. In constitutive modeling, symmetry analysis lets one formulate the response of a material in terms of so-called master curves, and construct rheological models based on a limited number of measurements. The application of symmetry methods leads to model reduction in a double sense: in treating large amounts number of measurements data to reduce them in a form exploitable for the construction of constitutive models, and by exploiting equivalence transformations extending point symmetries to efficiently reduce the number of significant parameters, and thus the computational cost of solving boundary value problems (BVPs). The symmetry framework and related conservation law analysis provide invariance properties of the constitutive models, allowing to predict the influence of a variation of the model parameters on the material response or on the solution of BVPs posed over spatial domains. The first part of the paper is devoted to the presentation of the general methodology proposed in this contribution. Examples of construction of rheological models based on experimental data are given for setting up a reduced model of the uniaxial creep and rupture behaviour of a Chrome-Molybdenum alloy $(9 \mathrm{Cr} 1 \mathrm{Mo})$ at different temperatures and stress levels. Constitutive equations for creep and rupture master responses are identified for this alloy, and validated based on experimental data. Equivalence transformations are exemplified in the context of parameter reduction in fully nonlinear anisotropic fiber-reinforced elastic solids.
\end{abstract}

Keywords: Experimental data, Symmetry and similarity methods, Constitutive modeling, Master Curves, Equivalence transformations, Creep and rupture invariants, Time-temperature equivalence, model reduction

\section{Introduction}

The notion of symmetry is a fundamental scientific concept present in many disciplines, from biology to mathematics and theoretical physics. Independently from its aesthetic

(c) The Author(s) 2021. This article is licensed under a Creative Commons Attribution 4.0 International License, which permits use, sharing, adaptation, distribution and reproduction in any medium or format, as long as you give appropriate credit to the original author(s) and the source, provide a link to the Creative Commons licence, and indicate if changes were made. The images or other third party material in this article are included in the article's Creative Commons licence, unless indicated otherwise in a credit line to the material. If material is not included in the article's Creative Commons licence and your intended use is not permitted by statutory regulation or exceeds the permitted use, you will need to obtain permission directly from the copyright holder. To view a copy of this licence, visit http://creativecommons.org/licenses/by/4.0/. 
attraction, the symmetry intrinsically contained in physical systems allows to concisely describe their invariance properties, classify and synthesize their mathematical structure and physical properties, and systematically derive exact solutions. Invariance properties of physical systems described by algebraic or differential equations emerge from these symmetries, including scale transformations, time, parity and charge inversions, gauge symmetries, dynamical symmetries, nonlocal symmetries.

Generalizing the discrete symmetry classification of crystals ( [1-3]), the notion of continuous symmetries, in particular, Lie groups of point symmetries introduced by Sophus Lie (e.g., [4]), with multiple further generalizations (e.g., [5,6] and references therein). Noether [7] provided a connection between conservation laws (energy, momentum, etc.) and local symmetries for models arising from a variational principle (see, e.g, [5,6]). The majority of field theories built in the twentieth century heavily rely on Lie symmetries, which are reflected in the form taken by the Lagrangian function [8]. In continuum mechanics, symmetries play a major role in the construction of the constitutive laws of materials, formulated from tensor valued functionals, objective (invariance of the material's response under the group of rotations in the three dimensional Euclidean space) and invariant under the action of the material symmetry group (discrete symmetries) $[9,10]$. The thermodynamics of irreversible processes (abbreviated as TIP in the sequel) is the natural framework for writing rheological models of dissipative and non dissipative materials, relying on thermodynamic potentials - namely the free energy and a dissipation or pseudo-dissipation potential. These potentials have to reflect the symmetry properties of the material being analyzed. Thereby, our approach relies on the postulate that the mechanical response of materials is associated with hidden symmetries, and our objective is precisely to reveal those symmetries from a set of measurements, and to write appropriate constitutive laws from them.

The consideration of Lie symmetries and equivalence transformations in the mechanics of materials is an emergent topic in the literature [11]. Recent works from the literature underline the interest of using Lie symmetries to obtain invariance properties of materials relying on experimental data; furthermore, Lie symmetries is a predictive methodology to predict the response of materials when the parameters inherent to their constitutive law change.

Invariance relations, together with master curves that constitute their graphical representation, are currently used in experimental rheology to synthesize the constitutive response of various materials subject to thermomechanical loadings. A typical example of such an empirical construction is the well-known time-temperature equivalence principle, stating an equivalence between the effect of time and of temperature, as proposed originally by Williams, Landel and Ferry in the so-called WLF model. In the same spirit, power laws in fatigue have been formulated in [12,13] (also [14] and references therein), using arguments of dimensional analysis in combination with similarity principles. These relationships are of a high practical interest, and it the aim of this contribution to highlight Lie symmetries and equivalence transformations as the underlying mathematical background.

From the point of view of solutions of the mechanical problem formulated in terms of differential equations, a symmetry is any transformation preserving the solution set, i.e., mapping solutions into solutions. In particular, solutions that are mapped into themselves, called the invariant solutions, can be algorithmically computed, and often have a clear 
physical meaning. This includes, for example, traveling waves and self-similar (scalinginvariant) solutions. While the full set of symmetries admitted by a model is a rather general geometrical object, Lie groups of point symmetries can be calculated using the local form of these symmetries, in terms of symmetry generators [5,6]. From a geometrical point of view, finding the symmetries of a given BVP allows to define a surface in the extended space of variables and parameters (when considering equivalence transformations), on which the solution is living.

The majority of contemporary mathematical models, in particular, physical models written in terms of partial or ordinary differential equations (PDEs, ODEs), involve constant or variable parameters. These parameters, also called arbitrary or constitutive elements, appear in the equations in the form of arbitrary constants (constitutive parameters) that assume values in certain ranges, and/or arbitrary functions (constitutive functions) belonging to certain classes. Equivalent formulations of a model at hand, involving fewer constitutive parameters and/or reduced forms of constitutive functions, can lead to a significant simplification of the analysis of the model, in particular, when such analysis involves classifications, such as local symmetry or conservation law classifications. Equivalent formulations of a class of models may be systematically sought using equivalence transformations $[15,16]$. Equivalence transformations are used to simplify classifications of mathematical models, relate models with seemingly different forms of constitutive functions and/or parameters, and simplify families of models by reducing the forms and number of such constitutive functions and/or parameters.

Lie symmetries, equivalence transformations, conservation laws, and other related analytical properties of nonlinear equations (cf. [6]) can be efficiently computed and classified using symbolic software, for example, the GeM package for Maple [17-19]. In particular, GeM can be used for systematic computation of equivalence transformations [16].

Another approach in constitutive modeling that is related to symmetry ideas, both philosophically and mathematically, is the use of entropy principles to predict and reduce the dependencies of constitutive functions. The approach consists in requiring that the admissible processes correspond to nonnegative entropy production, and yields constraints on the general postulated forms of constitutive functions. This constitutive modeling idea has been introduced pioneered by Ingo Müller's [20-24], and subsequently modified by Liu [25]; the result was called the Müller-Liu procedure. In the recent work [26,27], the entropy principle methodology was reformulated in terms of the solution set of the model equations, and a systematic procedure was suggested to use leading derivatives to compute constitutive function constraints; the approach was implemented in Maple symbolic software using the capabilities of the GeM package [27].

Recall that two main classes of approaches combining Lie symmetry metdhos and constitutive modeling have arisen in the literature, which will be referred to as the direct and the inverse problem or method, in the vocabulary used in [28]. The direct problem consists in finding Lie symmetries of a given set of constitutive equations, as developed by several authors in the field of mechanics of materials (see, e.g., [29,30]). It allows a priori the calculation of all symmetries and associated invariances of those equations, which may lead to geometrical transformation rules revealing (graphical) superpositions of the material responses (the superposition of those responses in a given space of variables gives the master curve). The methodology at the root of this construction consists of three main steps, which entail an algorithm for the determination of the constitutive law and 
master curves. In the first step, one looks for one or several Lie symmetries formulated from experimental master curves. Next, the subsequent symmetry conditions are applied to a general expression of the constitutive equations involving some unknown functions, resulting in a PDE system satisfied by these unknown functions. The comparison of the constructed constitutive model with experimental results allows to validate or refine the model if needed. As a last step, the inverse problem is linked to the direct problem: once a possible material constitutive law has been constructed, the material's response can be predicted outside the range of variation of the control variables involved in the measurements for varying experimental conditions, hence using Lie symmetries inherent to the obtained constitutive law as an extrapolation technique. Thereby, Lie symmetries leads to reduced constitutive models based on invariants having a true physical meaning, allowing to efficiently predict the response of materials with a complex rheological behavior when parameters change.

The paper is organized as follows. Section 2 describes in a synthetic manner the methodology of the application of Lie groups of symmetries to construct and simplify constitutive models. Section 3 presents an example of a computation of master curves and invariant constitutive equations modeling the creep and rupture behavior of metallic alloys. In Section 4, invariance relations and master responses associated to a rheological model are discussed. An example of the computation of equivalence transformations and parameter reduction in a model of nonlinear S-waves propagating in a hyperelastic fiber-reinforced material is presented in Section 5. The paper is concluded with a discussion in Section 6.

\section{Methodology}

For physical continuum systems, in particular, ones involving the mechanics of solids and fluids, the governing balance/evolution equations, as well as constitutive/rheological models, are commonly described by a set of algebraic and/or differential equations (ordinary, ODE, or partial, PDE). Symmetry ideas provide insights and rational methodologies to describe and study such models, revealing their intrinsic structure. For an experimental data set, the use of Lie group ideas is therefore not a simple interpolation of data, since the constitutive laws that can be constructed from a set of data based on a postulated Lie group structure allow both to interpolate within the data set and to extrapolate beyond the experimental parameter domains used to build the constitutive model.

The use of Lie groups in combination with experimentally recorded responses of a given material to formulate its constitutive law has been initiated in [11] and [31] (see also $[26,27]$. The aim of this contribution is to provide an overview of the Lie symmetry method in constitutive modeling. The generalized methodology proposed in this contribution is organized into the following steps:

- Formulation of Lie symmetries from measurements;

- Formulation of constitutive equations in terms of invariants;

- Computation of the Lie algebra and predictions of the material response for a range of variation of the parameters;

- Computation of equivalence transformations of constitutive models;

- Model simplification based on its admitted equivalence transformations. 
In subsequent developments, the above methodology is used to construct constitutive models describing the creep and rupture behavior of metallic alloys, both phenomena being highly stress- and temperature-dependent. In order to set the stage, we provide the non specialist with a comprehensive and synthetic overview of Lie symmetries, that will prove useful in subsequent developments.

\section{Lie symmetries: a basic summary}

Suppose a mathematical model formulated as a system of ordinary and/or partial differential equations $\mathcal{R}$

$$
R^{\sigma}(\boldsymbol{x}, \boldsymbol{u})=0, \quad \sigma=1, \ldots, N,
$$

of differential order $k \geq 1$, with $s \geq 1$ independent variables $\boldsymbol{x}=\left(x_{1}, \ldots, x_{s}\right)$ and $n \geq 1$ dependent variables $\boldsymbol{u}(\boldsymbol{x})=\left(u_{1}(\boldsymbol{x}), \ldots, u_{n}(\boldsymbol{x})\right)$. For brevity, we will denote any derivatives of dependent variables $\boldsymbol{u}(\boldsymbol{x})$ by subscript symbols

$$
\frac{\partial^{p} u_{v}(x)}{\partial x_{i_{1}} \ldots \partial x_{i_{p}}} \equiv\left(u_{v}\right)_{i_{1} \ldots i_{p}} .
$$

For the independent and dependent variables $\boldsymbol{x}, \boldsymbol{u}(\boldsymbol{x})$, one can consider the jet space $J^{q}(\boldsymbol{x} \mid \boldsymbol{u})$ : a multi-dimensional space of variables $\boldsymbol{x}, \boldsymbol{u}$, and all derivatives of $\boldsymbol{u}$ up to and including the order $q \geq k$. Then the DEs (2.1) (and possibly their differential consequences), treated as algebraic equations in $J^{q}(\boldsymbol{x} \mid \boldsymbol{u})$, define a manifold $\mathcal{S}$ in that space. This is the solution manifold of the system (2.1); indeed, any solution of (2.1) $\boldsymbol{u}(\boldsymbol{x})$ and its derivatives, for all admissible $x$, define a point on $\mathcal{S}$.

Lie groups of point transformations provide a mathematically sound tool to deform the solution manifold $\mathcal{S}$ of a given DE system to map its solutions to solutions of some other model; Lie point transformations preserving $\mathcal{S}$ yield Lie point symmetries of a given system (2.1). The admissible symmetry groups contain coordinate-independent, geometrical information about the solution set of a given model, enabling one to systematically construct exact invariant solutions and conservation laws, map known solutions and conservation laws to new ones, etc. (see, e.g., [6] for more details). vast literature is available on the topic, ranging from introductory and practically-oriented textbooks to more mathematically advanced and specialized (see, e.g., [5,6,32] and references therein). We now summarize the basic points required for the subsequent presentation.

A Lie group of point symmetries of a DE system (2.1) is a group of transformations of dependent and independent variables

$$
\begin{aligned}
& \bar{x}_{i}=f_{i}(\boldsymbol{x}, \boldsymbol{u} ; \mu)=x_{i}+\mu \xi^{i}(\boldsymbol{x}, \boldsymbol{u})+O\left(\mu^{2}\right), \quad i=1, \ldots, s, \\
& \bar{u}_{v}=g_{v}(\boldsymbol{x}, \boldsymbol{u} ; \mu)=u_{v}+\mu \eta^{v}(\boldsymbol{x}, \boldsymbol{u})+O\left(\mu^{2}\right), \quad v=1, \ldots, n,
\end{aligned}
$$

additive in the continuous parameter $\mu$, with the property that if (2.1) is a solution of $\boldsymbol{u}(\boldsymbol{x})$, then so is the transformed function $\overline{\boldsymbol{u}}(\overline{\boldsymbol{x}})$. For example, the scaling transformation

$$
\begin{aligned}
& \bar{x}=e^{\mu} x=x+\mu x+O\left(\mu^{2}\right), \\
& \bar{t}=e^{2 \mu} t=t+2 \mu t+O\left(\mu^{2}\right), \\
& \bar{u}=u
\end{aligned}
$$

leaves invariant the diffusion equation $u_{t}=u_{x x}$, and maps its every solution $u=f(x, t)$ into a new solution $\bar{u}=f\left(e^{-\mu} \bar{x}, e^{-2 \mu} \bar{t}\right)$, or removing bars and changing the sign of the parameter, simply into a solution $u_{1}(x, t)=f\left(e^{\mu} x, e^{2 \mu} t\right)$ with re-scaled space and time dependence. 
To every one-parameter Lie group of point transformations (2.2) there corresponds a unique infinitesimal generator (also known as the tangent vector field to the solution manifold of the system in the $(\boldsymbol{x}, \boldsymbol{u})$-space), given by

$$
\boldsymbol{v}=\xi^{i} \frac{\partial}{\partial x_{i}}+\eta^{v} \frac{\partial}{\partial u_{v}}
$$

(here and below, summation in repeated indices is assumed). For example, for the scaling transformation (2.3), one has

$$
\boldsymbol{v}=x \frac{\partial}{\partial x}+2 t \frac{\partial}{\partial t}+0 \cdot \frac{\partial}{\partial u} \text {. }
$$

The local generator (2.4), in turn, uniquely determines the global transformation (2.2), through the solution of an ODE system initial-value problem

$$
\begin{aligned}
& \frac{d \bar{x}_{i}}{d \mu}=\xi^{i}(\overline{\boldsymbol{x}}, \overline{\boldsymbol{u}}),\left.\quad \bar{x}_{i}\right|_{\mu=0}=x_{i}, \quad i=1, \ldots, s, \\
& \frac{d \bar{u}_{v}}{d \mu}=\eta^{v}(\overline{\boldsymbol{x}}, \overline{\boldsymbol{u}}),\left.\quad \bar{u}_{v}\right|_{\mu=0}=u_{v}, \quad v=1, \ldots, n,
\end{aligned}
$$

or briefly in the operator exponent notation,

$$
\bar{x}_{i}=e^{\mu v} x_{i}, \quad \bar{u}_{v}=e^{\mu v} u_{v} .
$$

A one-parameter Lie group of point transformations (2.2) induces one-parameter Lie groups of point transformations acting on the derivatives of $\boldsymbol{u}(\boldsymbol{x})$ in the jet space $J^{q}(\boldsymbol{x} \mid \boldsymbol{u})$, as follows:

$$
\begin{aligned}
& \left(\bar{u}_{v}\right)_{i}=u_{i}^{v}+\mu \eta_{i}^{(1) v}+O\left(\mu^{2}\right), \\
& \vdots \\
& \quad\left(\bar{u}_{v}\right)_{i_{1} \ldots i_{q}}=\left(u_{v}\right)_{i_{1} \ldots i_{q}}+\mu \eta_{i_{1} \ldots i_{q}}^{(q) v}+O\left(\mu^{2}\right) ;
\end{aligned}
$$

here the extended infinitesimals given by

$$
\begin{aligned}
& \eta_{i}^{(1) v}=\mathrm{D}_{i} \eta^{v}-\left(\mathrm{D}_{i} \xi^{j}\right) u_{j}^{v}, \\
& \eta_{i_{1} \ldots i_{k}}^{(k) v}=\mathrm{D}_{i_{k}} \eta_{i_{1} \ldots i_{k-1}}^{(k-1) v}-\left(\mathrm{D}_{i_{k}} \xi^{j}\right) u_{i_{1} \ldots i_{k-1} j}^{\mu}
\end{aligned}
$$

with $v=1, \ldots, n, i, i_{j}=1, \ldots, s$ for $j=1, \ldots, k$ with $k=2,3, \ldots, q$. The $q^{\text {th }}$ prolongation of the transformation generator (2.4) is given by

$$
\boldsymbol{v}^{(q)}=\xi^{i} \frac{\partial}{\partial x_{i}}+\eta^{v} \frac{\partial}{\partial u_{v}}+\eta_{i}^{(1) v} \frac{\partial}{\partial u_{i}^{v}}+\ldots+\eta_{i_{1} \ldots i_{q}}^{(q) v} \frac{\partial}{\partial\left(u_{v}\right)_{i_{1} \ldots i_{q}}} .
$$

The main mathematical tool used to systematically seek Lie groups of point transformations (2.2) that are symmetries of a DE system (2.1) is the infinitesimal invariance condition

$$
v^{(k)} R^{\alpha}\left(x, u, \partial u, \ldots, \partial^{k} u\right)=0
$$

holding on all solutions of (2.1). The invariance condition expresses the fact that the mapping generated by the transformation is locally tangent to the solution set of the DE system (2.1), and upon splitting, yields a set of linear determining PDEs that define the unknown symmetry components $\xi^{i}, \eta^{v}$ of admissible symmetry generators (2.4) (e.g., $[5,6])$. The substitution of solution space conditions and splitting of the determining equations can often be efficiently accomplished in symbolic software, such as the GeM package for Maple [17-19]. 
Another important notion related to Lie symmetries of differential equations, or more generally, to Lie group of point transformations, is that of an invariant, or a differential invariant. A function $F(\boldsymbol{x}, \boldsymbol{u})$ is called invariant under the group of transformations (2.2) when

$$
F(\overline{\boldsymbol{x}}, \overline{\boldsymbol{u}})=F(\boldsymbol{x}, \boldsymbol{u}) .
$$

Locally, the invariance condition is given by the vanishing action of the transformation generator (2.4), as a differential operator, on $F$ :

$$
\boldsymbol{v} F(\boldsymbol{x}, \boldsymbol{u})=0 .
$$

One of the simplest methods to find invariants of a given one-parameter Lie group of transformations (2.2) is through the solution of the characteristic system

$$
\frac{d x_{1}}{\xi^{1}}=\ldots=\frac{d x_{s}}{\xi^{s}}=\frac{d u_{1}}{\eta^{1}}=\ldots=\frac{d u_{n}}{\eta^{n}}
$$

yielding a solution that can be written in the form

$$
I^{1}(\boldsymbol{x}, \boldsymbol{u})=c_{1}, \ldots, I^{n+s-1}(\boldsymbol{x}, \boldsymbol{u})=c_{n+s-1},
$$

where $c_{i}$ are arbitrary integration constants, and $I^{i}$ are $n+s-1$ functionally independent functions that do not involve any free integration constants. The functions $\left\{I^{i}\right\}$ are the elementary transformation invariants; more general invariants can be obtained as arbitrary functions of $\left\{I^{i}\right\}$.

Seeking invariants (2.11) forms an essential part of the procedure of seeking canonical coordinates that are used to produce symmetry-reduced versions of physical models (e.g., $[5,6])$.

In a fashion similar to that for invariant functions, differential invariants of the group of transformations (2.2) $F[\boldsymbol{u}]$, differential functions that may depend on $\boldsymbol{x}, \boldsymbol{u}$, and derivatives of $\boldsymbol{u}$ to some order $k$, satisfy the invariance condition

$$
\boldsymbol{v}^{(k)} F[\boldsymbol{u}]=0 .
$$

As an example, the scaling transformation (2.3) has invariants arising from solving the system

$$
\frac{d x}{x}=\frac{d t}{2 t}=\frac{d u}{0}
$$

for example, $I^{1}=x / \sqrt{t}, I^{2}=u$, which allows one to seek symmetry-invariant solutions of the diffusion equation in the form $u(x, t)=f(x / \sqrt{t})$. Differential invariants corresponding to the scaling transformation (2.3) include $x^{2} u_{t}, u_{t} / u_{x x}, u_{t t} / u_{x x x x}$, etc.

\section{Equivalence transformations and their computation}

In many cases, it is important to consider a class of mathematical models consisting of governing dynamic equations and constitutive relations as a whole. Such models are often given by systems of ordinary and/or partial differential equations, and/or algebraic equations, forming a family $\mathcal{R}_{p}$

$$
R^{\sigma}(\boldsymbol{x}, \boldsymbol{u}, \boldsymbol{p})=0, \quad \sigma=1, \ldots, N,
$$

involving $s$ independent variables $\boldsymbol{x}=\left(x_{1}, \ldots, x_{s}\right), n$ dependent variables $\boldsymbol{u}(\boldsymbol{x})=$ $\left(u_{1}(\boldsymbol{x}), \ldots, u_{n}(\boldsymbol{x})\right)$, and $m$ arbitrary elements (constitutive functions and/or parameters) 
$\boldsymbol{p}=\left(p_{1}, \ldots, p_{m}\right)$. The expressions $R^{\sigma}$ in (2.12) may involve derivatives of $\boldsymbol{u}$ and functions within $\boldsymbol{p}$ as necessary.

An equivalence transformation for a DE family $\mathcal{R}_{\boldsymbol{p}}$ is commonly defined as a change of variables and arbitrary elements

$$
(\boldsymbol{x}, \boldsymbol{u}, \boldsymbol{p}) \rightarrow(\overline{\boldsymbol{x}}, \overline{\boldsymbol{u}}, \overline{\boldsymbol{p}})
$$

that maps every system of equations (2.12) into a system of equations in the same family $\mathcal{R}_{\boldsymbol{p}}$ (see, e.g., [6,16] and references therein).

Generally, no systematic procedure exists to compute explicit formulas of the transformations (2.13). However, equivalence transformations that form Lie groups, such as point equivalence transformations

$$
\begin{aligned}
& \bar{x}_{i}=f_{i}(\boldsymbol{x}, \boldsymbol{u} ; \mu)=x_{i}+\mu \xi^{i}(\boldsymbol{x}, \boldsymbol{u})+O\left(\mu^{2}\right), \quad i=1, \ldots, s, \\
& \bar{u}_{\nu}=g_{\nu}(\boldsymbol{x}, \boldsymbol{u} ; \mu)=u_{\nu}+\mu \eta^{v}(\boldsymbol{x}, \boldsymbol{u})+O\left(\mu^{2}\right), \quad v=1, \ldots, n, \\
& \bar{p}_{\lambda}=G_{\lambda}\left(\boldsymbol{Q}_{\lambda} ; \mu\right)=p_{\lambda}+\mu \theta^{\lambda}\left(\boldsymbol{Q}_{\lambda}\right)+O\left(\mu^{2}\right), \quad \lambda=1, \ldots, m,
\end{aligned}
$$

can be computed systematically, and therefore are commonly used in practice $[15,16,33]$. In (2.14), the form of the argument $\boldsymbol{Q}_{\lambda}$ of the transformation $G_{\lambda}$ and the tangent vector field component $\theta^{\lambda}$ depends on the nature of the arbitrary element $p_{\lambda}, \lambda=1, \ldots, m$. For example, if $p_{\ell}$ is a constitutive parameter, $\boldsymbol{Q}_{\lambda}$ may be a set of constitutive parameters of the model; if $p_{\lambda}$ is a constitutive function, then $\boldsymbol{Q}_{\lambda}$ may involve quantities that $p_{\lambda}$ depends on, and/or other constitutive functions with compatible dependencies. In the above definition, the transformations form a group on the $(\boldsymbol{x}, \boldsymbol{u})$ space; in particular, the transformations for $\boldsymbol{x}, \boldsymbol{u}$ do not involve arbitrary elements. The infinitesimal generator for (2.14) takes the form

$$
\boldsymbol{v}=\xi^{i} \frac{\partial}{\partial x_{i}}+\eta^{\nu} \frac{\partial}{\partial u_{v}}+\theta^{\lambda} \frac{\partial}{\partial p_{\lambda}} .
$$

Lie groups of equivalence transformations (2.14) are closely related to Lie groups (2.2) of point symmetries mapping the model equations (2.12) into themselves, without change of the parameters [5,6].

As an elementary example of equivalence transformations (2.14), one can consider the non-dimensionalization of (1+1)-dimensional linear linear wave equation

$$
u_{t t}=c^{2} u_{x x}, \quad c=\text { const, }
$$

for the unknown $u(x, t)$. It is evident that the PDE family (2.16) clearly admits scaling and translation-type equivalence transformations

$$
\bar{x}=A_{3} x+A_{1}, \quad \bar{t}=A_{4} t+A_{2}, \quad \bar{u}(\bar{x}, \bar{t})=A_{5} u(x, t), \quad \bar{c}=\frac{A_{3}}{A_{4}} c,
$$

where $A_{1}, \ldots, A_{5}$ are arbitrary nonzero constants. Indeed, if $u(x, t)$ is a solution of the PDE (2.16) with a constitutive parameter $c$, then $\bar{u}(\bar{x}, \bar{t})$ is a solution of a PDE

$$
\bar{u}_{\bar{t} \bar{t}}=\bar{c}^{2} \bar{u}_{\bar{x} \bar{x}}
$$

a member of the family (2.16) with a different constitutive parameter, $\bar{c}$. In particular, if $A_{3}=L$ and $A_{5}=u_{0}$ are the characteristic size and wave amplitude, then one can choose $A_{4}=A_{3} c$ as the characteristic time of the model, and the PDE (2.18) will become

$$
\bar{u}_{\bar{t} \bar{t}}=\bar{u}_{\bar{x} \bar{x}},
$$


that is, dimensionless, and involving no parameters. This is the basic idea of parameter reduction; similarly, when constitutive functions are involved, their forms may be simplified using equivalence transformations. We note that in the form of Lie groups, the transformations (2.17) include five one-parameter groups of equivalence transformations

$$
x^{*}=e^{\mu_{3}} x+\mu_{1}, \quad t^{*}=e^{\mu_{4}} t+\mu_{2}, \quad u^{*}\left(x^{*}, t^{*}\right)=e^{\mu_{5}} u(x, t), \quad c^{*}\left(u^{*}\right)=e^{\mu_{3}-\mu_{4}} c(u),
$$

with group parameters $\mu_{i}, i=1, \ldots, 5$, and infinitesimal generators

$$
\boldsymbol{v}_{1}=\frac{\partial}{\partial x}, \quad \boldsymbol{v}_{2}=\frac{\partial}{\partial t}, \quad \boldsymbol{v}_{3}=x \frac{\partial}{\partial x}+c \frac{\partial}{\partial c}, \quad \boldsymbol{v}_{4}=t \frac{\partial}{\partial t}-c \frac{\partial}{\partial c}, \quad \boldsymbol{v}_{5}=u \frac{\partial}{\partial u} .
$$

Various extensions of the notion of equivalence transformations (2.14) exist (see, e.g., [16] for a brief review). A natural extension arises when the transformation components for the independent and/or dependent variables $\xi^{i}, \eta^{v}$ are allowed to additionally depend on arbitrary elements:

$$
\boldsymbol{v}=\xi^{i}(\boldsymbol{x}, \boldsymbol{u}, \boldsymbol{p}) \frac{\partial}{\partial x_{i}}+\eta^{v}(\boldsymbol{x}, \boldsymbol{u}, \boldsymbol{p}) \frac{\partial}{\partial u_{v}}+\theta^{\lambda}(\boldsymbol{x}, \boldsymbol{u}, \boldsymbol{p}) \frac{\partial}{\partial p_{\lambda}} .
$$

These are referred to as groups of generalized equivalence transformations $[15,34,35]$.

Lie groups of generalized equivalence transformations for a family of models $\mathcal{R}_{\boldsymbol{p}}$ (2.12) can be constructed in the following manner. (Here for simplicity we assume that if the set $\boldsymbol{p}$ involves arbitrary functions, these functions do not depend on derivatives of $\boldsymbol{u}$.)

1. Replace the constitutive functions and/or parameters $\boldsymbol{p}$ by new dependent variables $\left(p_{1}(\boldsymbol{x}), \ldots, p_{m}(\boldsymbol{x})\right)$. Thus consider a new model given by a system of equations $\widetilde{\mathcal{R}}$ with $n+m$ dependent variables and no arbitrary elements.

2. Seek point symmetries of $\widetilde{\mathcal{R}}$, with infinitesimal generators (2.21). Obtain the split system of determining equations for the unknown symmetry generator components $\xi^{i}(\boldsymbol{x}, \boldsymbol{u}, \boldsymbol{p}), \eta^{\nu}(\boldsymbol{x}, \boldsymbol{u}, \boldsymbol{p}), \theta^{\lambda}(\boldsymbol{x}, \boldsymbol{u}, \boldsymbol{p})$.

3. If the arbitrary elements $\boldsymbol{p}$ of the original model (2.12) contained arbitrary functions, introduce restrictions of the form

$$
\frac{\partial \xi^{i}(\boldsymbol{x}, \boldsymbol{u}, \boldsymbol{p})}{\partial p_{\gamma}}=0, \quad \frac{\partial \eta^{\nu}(\boldsymbol{x}, \boldsymbol{u}, \boldsymbol{p})}{\partial p_{\delta}}=0, \quad \frac{\partial \theta^{\lambda}(\boldsymbol{x}, \boldsymbol{u}, \boldsymbol{p})}{\partial x_{j}}=0,
$$

etc., as appropriate, to exclude the dependence of transformation components of the arbitrary elements on variables they do not depend on, as well as the dependence of transformation components of the variables of the system on inappropriate arbitrary elements. For example, for the DE family (2.16), the infinitesimal generator of the generalized equivalence transformations has the form

$$
\boldsymbol{v}=\xi(x, t, u, c) \frac{\partial}{\partial x}+\tau(x, t, u, c) \frac{\partial}{\partial t}+\eta(x, t, u, c) \frac{\partial}{\partial u}+\theta(x, t, u, c) \frac{\partial}{\partial c},
$$

and since $c=$ const, the transformation for $c$ must not explicitly depend on the variables $x, t, u$. Therefore the restrictions on the component $\theta$ are given by

$$
\frac{\partial \theta(x, t, u, c)}{\partial x}=\frac{\partial \theta(x, t, u, c)}{\partial t}=0=\frac{\partial \theta(x, t, u, c)}{\partial u}=0 .
$$

4. In order to simplify computations, additional restrictions can be introduced at this stage, for example,

$$
\frac{\partial \xi^{i}(x, u, K)}{\partial p_{\gamma}}=0, \quad i=1, \ldots, s, \quad \gamma=1, \ldots, m,
$$


if the transformations for the independent variables are assumed to be independent of the arbitrary elements.

5. Append all restrictions, in the form of linear PDEs, to the split system of determining equations.

6. Simplify and solve the augmented split system of determining equations, to find the infinitesimal generators $\boldsymbol{v}$ (2.15) of the generalized equivalence transformations.

7. Integrate to obtain the global group (2.14) of generalized equivalence transformations

$$
\begin{aligned}
& \bar{x}_{i}=f_{i}(\boldsymbol{x}, \boldsymbol{u}, \boldsymbol{p} ; \mu), \quad i=1, \ldots, s, \\
& \bar{u}_{\mu}=g_{\mu}(\boldsymbol{x}, \boldsymbol{u}, \boldsymbol{p} ; \mu), \quad \mu=1, \ldots, n, \\
& \bar{p}_{\ell}=G_{\ell}(\boldsymbol{x}, \boldsymbol{u}, \boldsymbol{p} ; \mu), \quad \ell=1, \ldots, m .
\end{aligned}
$$

Specifically, for each infinitesimal generator (2.15), the corresponding one-parameter Lie group of equivalence transformations is found through the solution of the initialvalue problem

$$
\begin{aligned}
& \frac{d}{d \mu} \bar{x}_{i}=\xi^{i}(\overline{\boldsymbol{x}}, \overline{\boldsymbol{u}}, \overline{\boldsymbol{p}}),\left.\quad \bar{x}_{i}\right|_{\mu=0}=x_{i}, \quad i=1, \ldots, s, \\
& \frac{d}{d \mu} \bar{u}_{\nu}=\eta^{\nu}(\overline{\boldsymbol{x}}, \overline{\boldsymbol{u}}, \overline{\boldsymbol{p}}),\left.\quad \bar{u}_{\nu}\right|_{\mu=0}=u_{\nu}, \quad v=1, \ldots, n, \\
& \frac{d}{d \mu} \bar{p}_{\lambda}=\theta^{\lambda}(\overline{\boldsymbol{x}}, \overline{\boldsymbol{u}}, \overline{\boldsymbol{p}}),\left.\quad \bar{p}_{\lambda}\right|_{\mu=0}=p_{\lambda}, \quad \lambda=1, \ldots, m .
\end{aligned}
$$

where $\mu$ is the group parameter.

After the equivalence transformation (2.26) have been found, they can be used to observe which forms of the arbitrary elements (constitutive functions and/or parameters) $\boldsymbol{p}$ can be related to other sets of arbitrary elements $\overline{\boldsymbol{p}}$; such sets of related arbitrary elements can be treated as one for all solution and classification purposes. In particular, the set $\overline{\boldsymbol{p}}$ can be chosen to eliminate or simplify the arbitrary element set $\boldsymbol{p}$; as a result, one may need to deal with a simpler model (e.g., a simpler PDE boundary value problem) than the originally posed one.

The above procedure is readily generalized to the cases when the given model involves arbitrary functions and their derivatives, and/or arbitrary functions that depend on more complex combinations of independent and dependent variables and/or derivatives of dependent variables of the model. The latter, for example, is a common case for the stored mechanical energy density function in hyperelasticity theory.

The construction of invariance relations is illustrated in the next section to construct constitutive models for the creep and rupture behavior of metallic alloys. An example of a computation of equivalence transformations for a family of nonlinear wave equations, and the resulting significant simplification of the model using parameter reduction, is presented in Section 5 below.

\section{Invariance relations for the creep and rupture behavior of metallic alloys}

One important field in which invariant relations are used is the high temperature creep of metallic alloys, devoted to industrial applications. Landmarks in this context are the Larson-Miller and the Dorn models (see e.g. [36] and the references therein). Those relations intrinsically have the meaning of invariants, and further allow to extrapolate experimental data at different temperatures, assuming that the microstructure of the material remains stable during the creep test. The two main objectives of this kind of 
investigation are either the determination of the time needed to reach a given strain for fixed stress and temperature, or the estimation of the time leading to rupture, in the same controlled conditions. In principle, both problems, although interrelated (viscoplasticity is coupled to damage), are treated separately, namely a creep problem, and damage leading to rupture. We consider in the present study the creep behaviour of the $9 \mathrm{Cr} 1 \mathrm{Mo}$ martensitic stainless steel, which is also known for its good thermal-fatigue strength and oxydation resistance ([37]).

According to experts in the field, one of the most crucial problem in determining the integrity of structural components is the creep behaviour of ferritic-martensitic steels used in supercritical plants at high temperature due to their reduced cost, lower thermal expansion coefficient, and better radiation stability combined with adequate corrosion resistance (see [38] and the references therein) [39]. We approach the creep and rupture behavior from a phenomenological standpoint, with the objective to construct constitutive laws for the creep and rupture of metallic alloys, relying on a combination of experimental data and symmetry analysis. We shall rely on experimental data of [40], obtained for the $9 \mathrm{Cr} 1 \mathrm{Mo}$ alloy, when submitted to creep tests carried out at different constant temperatures and different constant stress levels.

In the present case, the observable variables are the strain rate $u_{1}=\dot{\varepsilon}$ and the time to rupture $u_{2}=t_{R}$, while the control variables are the applied stress $p_{1}=\sigma$, the absolute temperature $p_{2}=T$, and the time $p_{3}=t$.

\section{Rupture behavior: master curves}

Isothermal rupture curves at different temperatures are first considered, and represented in the logarithmic plane (see Fig. 4). If we consider only "simple" geometrical mappings such as translations or homothetic mappings, it can be seen that a master curve can be obtained by shifting the curves along the $\log t_{R}$ and $\log \sigma$ axis, as shown in Fig. 5. From a mathematical point of view, one then looks for the following geometrical mapping:

$$
\left\{\begin{aligned}
\bar{T} & =T+\mu \\
\log \bar{t}_{R} & =a_{1}(\mu)+\log t_{R} \\
\log \bar{\sigma} & =b_{1}(\mu)+\log \sigma
\end{aligned}\right.
$$

with $a_{1}(\mu)$ and $b_{1}(\mu)$ the shift factors along the $\log t_{R}$ and $\log \sigma$ axis respectively. If the (arbitrary) chosen value of $\bar{T}$ is $748 \mathrm{~K}$, then $a_{1}(\mu)$ and $b_{1}(\mu)$ correspond to the shift required to move the curve at $T$ to make it coincide with those at $\bar{T}$, if $\mu$ is defined by $\bar{T}=T+\mu$. If we assume that Eqs. (3.1) define a Lie group, then the standard Lie group axioms have to be fulfilled (cf. [5]). It can be readily shown that they yield the following conditions to be satisfied by functions $a_{1}(\mu)$ and $b_{1}(\mu)$ :

$$
\left\{\begin{array}{rl}
a_{1}(0) & =0 \\
a_{1}(-\mu) & =-a_{1}(\mu) \\
a_{1}\left(\mu_{1}+\mu_{2}\right) & =a_{1}\left(\mu_{1}\right)+a_{1}\left(\mu_{2}\right)
\end{array},\left\{\begin{aligned}
b_{1}(0) & =0 \\
b_{1}(-\mu) & =-b_{1}(\mu) \\
b_{1}\left(\mu_{1}+\mu_{2}\right) & =b_{1}\left(\mu_{1}\right)+b_{1}\left(\mu_{2}\right)
\end{aligned}\right.\right.
$$

for all $\mu, \mu_{1}, \mu_{2}$ in $\mathbb{R}$. From Eqs. (3.2), one can infer the linearity of $a_{1}(\mu)$ and $b_{1}(\mu)$ with respect to $\mu$ :

$$
a_{1}(\mu)=\alpha \mu, \quad b_{1}(\mu)=\beta \mu,
$$

where $\alpha$ and $\beta$ are true constants. Those last relations are in great accordance with the experimental values of the shift factors. The two fits give the following values of the 
coefficients:

$$
\alpha=-0.0403636, \quad \beta=-0.00584727,
$$

that will be used here and in all subsequent developments. Hence, if one inserts (3.3) into (3.1), the following observed Lie group is obtained:

$$
G_{1}^{o b s}:\left\{\begin{array}{l}
\bar{T}=T+\mu \\
\bar{t}_{R}=e^{\alpha \mu} t_{R} \\
\bar{\sigma}=e^{\beta \mu} \sigma
\end{array}\right.
$$

and maps an experimental curve into another one. Of course, this Lie group is only valid for the explored values of $T$, but its validity is presently assumed for all $T$ between the extremal values $748 \mathrm{~K}$ and $923 \mathrm{~K}$. The components of the generator $\boldsymbol{v}_{1}^{\text {obs }}$ associated with $G_{1}^{o b s}$ are given by the derivation of Eqs. (3.5) with respect to $\mu$ at $\mu=0$, viz:

$$
\boldsymbol{v}_{1}^{o b s}=\alpha t_{R} \frac{\partial}{\partial t_{R}}+\beta \sigma \frac{\partial}{\partial \sigma}+\frac{\partial}{\partial T} .
$$

The Lie group generated by this vector field allows to shift all rupture responses in the same plane to the response obtained for a chosen reference temperature; the curve associated with this response is designated as the master curve. A similar strategy may be applied to the curves presented in Fig. 6, picturing the "isostrain" creep curves in the logarithmic plane $\log \sigma$ vs. $\log t$. As previously, one shall search the transformation rules:

$$
\left\{\begin{aligned}
\bar{\varepsilon} & =e^{\mu} \varepsilon \\
\log \bar{t} & =a_{2}(\mu)+\log t \\
\log \bar{\sigma} & =b_{2}(\mu)+\log \sigma
\end{aligned}\right.
$$

mapping a curve at $\varepsilon$ to a reference curve at $\bar{\varepsilon}=5 \%$ (observe that the chosen value of the reference strain is arbitrary). The obtained master curve is shown in Fig. 7. The axioms of Lie symmetries lead as in section 3.1 to the linearity of $a_{2}(\mu)$ and $b_{2}(\mu)$ with respect to $\mu$ :

$$
a_{2}(\mu)=\gamma \mu, \quad b_{2}(\mu)=\delta \mu,
$$

with $\gamma$ and $\delta$ new constants given by:

$$
\gamma=1.57167, \quad \delta=-0.0172415 \text {. }
$$

The calculated linear fit of $a_{2}(\mu)$ and $b_{2}(\mu)$ is validated in Fig. 8, and a second Lie group $G_{2}^{o b s}$ is obtained by inserting (3.8) into (3.7):

$$
G_{2}^{o b s}:\left\{\begin{aligned}
\bar{\varepsilon} & =e^{\mu} \varepsilon \\
\bar{t} & =e^{\gamma \mu} t \\
\bar{\sigma} & =e^{\delta \mu} \sigma .
\end{aligned}\right.
$$

The generator $\boldsymbol{v}_{2}^{o b s}$ of $G_{2}^{o b s}$ is given by:

$$
\boldsymbol{v}_{2}^{o b s}=\gamma t \frac{\partial}{\partial t}+\delta \sigma \frac{\partial}{\partial \sigma}+\varepsilon \frac{\partial}{\partial \varepsilon} \text {. }
$$

The prolongations of the vector fields $\boldsymbol{v}_{1}^{o b s}$ and $\boldsymbol{v}_{2}^{o b s}$ account for the component with respect to $\dot{\varepsilon}$ and shall prove useful in the sequel. They can be computed with the prolongation formulae ([5]):

$$
\begin{aligned}
& \operatorname{pr}^{(1)} \boldsymbol{v}_{1}^{o b s}=\alpha t_{R} \frac{\partial}{\partial t_{R}}+\beta \sigma \frac{\partial}{\partial \sigma}+\frac{\partial}{\partial T}, \\
& \operatorname{pr}^{(1)} \boldsymbol{v}_{2}^{o b s}=\gamma t \frac{\partial}{\partial t}+\delta \sigma \frac{\partial}{\partial \sigma}+\varepsilon \frac{\partial}{\partial \varepsilon}+\dot{\varepsilon}(1-\gamma) \frac{\partial}{\partial \dot{\varepsilon}} .
\end{aligned}
$$


At this stage, recall that two generators $\left(\boldsymbol{v}_{1}^{o b s}\right.$ and $\left.\boldsymbol{v}_{2}^{o b s}\right)$ have been identified from the experimental data set. We now look for a set of constitutive equations that can capture the creep behaviour of the material. The first constitutive relation expresses the rupture time $t_{R}$ versus the loading parameters ( $T$ and $\sigma$ in the present case) while the second one gives the evolution of the strain rate $\dot{\varepsilon}$ as a function of $t, \sigma, \varepsilon$, and $T$ :

$$
\begin{aligned}
& \Delta_{1}=t_{R}-f(\sigma, T)=0, \\
& \Delta_{2}=\dot{\varepsilon}-h(t, \sigma, \varepsilon, T)=0 .
\end{aligned}
$$

In those two equations, $h$ and $f$ are unknown functions that will be further determined. As concerns the classification of symmetries, it seems reasonable to apply the symmetry condition $\boldsymbol{v}_{1}^{o b s}$ to $\Delta_{1}$ and $\boldsymbol{\nu}_{2}^{o b s}$ to $\Delta_{2}$. Indeed, the generator $\boldsymbol{v}_{1}^{o b s}$ ( $\boldsymbol{v}_{2}^{o b s}$ respectively) has been built by only considering rupture curves (creep curves respectively). The satisfaction of those symmetry conditions means that each constitutive law (creep and rupture) keeps the same form and remains valid when the considered parameter changes. Finally, the complete set of symmetry conditions writes:

$$
\begin{aligned}
& \operatorname{pr}^{(1)} \boldsymbol{v}_{1}^{o b s} \Delta_{1}=0, \quad \text { whenever } \Delta_{1}=0, \\
& \operatorname{pr}^{(1)} \boldsymbol{v}_{2}^{o b s} \Delta_{2}=0, \quad \text { whenever } \Delta_{2}=0,
\end{aligned}
$$

and it is associated with the sets $K_{1}=\{1\}$ and $K_{2}=\{2\}$. The next step consists in writing the constitutive models in terms of invariants.

\section{Formulation of the constitutive equations for creep and rupture}

As $m_{1}=1$ and $m_{2}=1$, the methodologies described in section 2 can be applied. The first methodology (direct solving of the symmetry conditions) has already been applied in [11]; we shall here consider the strategy involving a combination of invariants, in the spirit of the extrapolation methods of the literature, see the review article [36] and references therein. Thus, one looks for two new functions $\Sigma_{1}\left(I_{1}, I_{2}\right)$ and $\Sigma_{2}\left(J_{1}, J_{2}, J_{3}\right)$ such that

$$
\begin{aligned}
\Sigma_{1}\left(I_{1}, I_{2}\right) & =0 \Leftrightarrow \Delta_{1}=0, \\
\Sigma_{2}\left(J_{1}, J_{2}, J_{3}\right) & =0 \Leftrightarrow \Delta_{2}=0,
\end{aligned}
$$

with $I_{1}, I_{2}$ the invariants of $\boldsymbol{v}_{1}^{o b s}$ given by the solution of the system

$$
\frac{d t_{R}}{\alpha t_{R}}=\frac{d \sigma}{\beta \sigma}=d T,
$$

and $J_{1}, J_{2}, J_{3}$ the invariants of $\boldsymbol{v}_{2}^{o b s}$ given by the solution of the same characteristic system:

$$
\frac{d t}{\gamma t}=\frac{d \sigma}{\delta \sigma}=\frac{d \varepsilon}{\varepsilon}=\frac{d \dot{\varepsilon}}{(1-\gamma) \dot{\varepsilon}} .
$$

Solving (3.19) and (3.20) gives the following invariants for rupture

$$
I_{1}=\log t_{R}-\alpha T, \quad I_{2}=\log \sigma-\beta T,
$$

and creep

$$
J_{1}=\log t-\gamma \log \varepsilon, \quad J_{2}=\log \sigma-\delta \log \varepsilon, \quad J_{3}=\log \dot{\varepsilon}-(1-\gamma) \log \varepsilon .
$$

Considering rupture, the experimental representation of $\log \left(t_{R}\right)$ vs. $1 / T$ pictured in Fig. 9 suggests a relation of the form:

$$
\log t_{R} \approx \frac{F_{1}(\sigma)}{T}+F_{2}(\sigma) .
$$


We here decide to keep the dependence of the slope $F_{1}$ with respect to $\sigma$, since the very low number of experimental points in Fig. 9 does not allow to assume a constant slope. To follow as closely as possible the mathematical structure of (3.23), the following expression is proposed:

$$
\Sigma_{1}\left(I_{1}, I_{2}\right)=\frac{K_{1}}{I_{2}}+K_{2} I_{1}+K_{3} I_{2}=0,
$$

in which $K_{1}, K_{2}, K_{3}$ are constants, which warrants a hyperbolic dependence with respect to $T$. Eq. (3.24) leads to:

$$
\log t_{R}=\frac{a}{\log \sigma-\beta T}+b \log \sigma+(\alpha-b \beta) T
$$

with $a=-K_{1} / K_{2}$ and $b=-K_{3} / K_{2}$ new constants. Eq. (3.25) shows that a linear term in $T$ appears by combining the invariants $I_{1}$ and $I_{2}$, giving a relation which slightly differs from the Larson-Miller or Dorn relationships. However, this linear term is necessary to fullfill the symmetry condition (3.15) that follows from the experimental data set. The validation of Eq. (3.25) is given in Fig. 10, in which a good agreement with the experimental data presented in [40] is observed (the coefficients $a$ and $b$ are adjusted). As to the creep strain rate, we introduce in accordance with the "convenient creep laws" of [40] the power function:

$$
\Sigma_{2}\left(J_{1}, J_{2}, J_{3}\right)=e^{J_{3}}-A e^{c J_{1}} e^{d J_{2}}=\dot{\varepsilon} \varepsilon^{-(1-\gamma)}-A t^{c} \varepsilon^{-c \gamma} \sigma^{d} \varepsilon^{-d \delta}=0
$$

with $A, c$ and $d$ some constants, which leads in turn to:

$$
\dot{\varepsilon}=A t^{c} \sigma^{d} \varepsilon^{1-\gamma-c \gamma-d \delta} \text {. }
$$

It can be easily shown that the symmetry conditions (3.15) and (3.16) are fulfilled. If we denote by (i) $\varepsilon(t=0, \sigma, T)=\varepsilon_{0}(\sigma, T)$ the initial value of the strain $\varepsilon$ and (ii) the exponent $v=\gamma+c \gamma+d \delta$, then the resolution of Eq. (3.27) allows to write:

$$
\varepsilon(t)=(1+c)^{-1 / v}\left[(1+c) \varepsilon_{0}(\sigma, T)^{v}+A v t^{c+1} \sigma^{d}\right]^{1 / v} .
$$

If the temperature $T$ and the strain $\varepsilon$ are assigned fixed values, and if we assume that the initial strain can be written in terms of a Hookean relation:

$$
\varepsilon_{0}(\sigma, T)=\frac{\sigma}{E(T)}
$$

with $E(T)$ the Young modulus at $T$, then the "isostrain" curves may be obtained by expressing the time $t(\sigma)$ from Eq. (3.28), viz:

$$
t(\sigma)=\left[\frac{(c+1)\left(\varepsilon^{v}-\sigma^{v} E(T)^{-v}\right)}{A v \sigma^{d}}\right]^{1 /(1+c)} .
$$

The inverse functional dependence $\sigma(t)$ can be numerically calculated. The validation of the model $\log \sigma$ vs. $\log t$ can then be carried out through Eq. (3.30), and is pictured in Fig. 11; the model and the experimental data are seen to be in good agreement (the coefficients $E(T), A$ and $c, d$ are here adjusted). Figure. 12 pictures the creep curves at different stress levels as predicted by the present model.

\section{Computation of the Lie algebra for creep and rupture}

To summarize the previous sections, the following constitutive equations have been obtained as representative of the creep and rupture behaviours of the considered $9 \mathrm{Cr} 1 \mathrm{Mo}$ stainless steel:

$$
\dot{\varepsilon}=A t^{c} \sigma^{d} \varepsilon^{1-\gamma-c \gamma-d \delta} \quad \text { if } t \leq t_{R},
$$


Table 1 Some creep invariants encountered in the literature (see, e.g., Ref. [61])

\begin{tabular}{ll}
\hline Authors & $\operatorname{lnvariant}(\mathrm{s})$ \\
\hline Larson \& Miller & $T\left(\log t+\log B_{2}\right)$ \\
Dorn & $\frac{B_{1}}{T}-\log t$ \\
Monkman-Grant & $\log t_{R}+m \log \dot{\varepsilon}_{\text {min }}$ \\
Modified Monkman-Grant & $\log \left(\frac{t_{R}}{\varepsilon_{R}}\right)+m^{\prime} \log \dot{\varepsilon}_{\text {min }}$ \\
\hline
\end{tabular}

Table 2 Commutator table of the Lie algebra of Eq. (3.31)

\begin{tabular}{lllll}
\hline & $\boldsymbol{v}_{2}^{1}$ & $\boldsymbol{v}_{2}^{2}$ & $\boldsymbol{v}_{2}^{3}$ & $\boldsymbol{v}_{2}^{4}$ \\
\hline $\boldsymbol{v}_{2}^{1}$ & 0 & 0 & $(c+1) \boldsymbol{v}_{2}^{1}$ & $2(c+1) \boldsymbol{v}_{2}^{1}$ \\
$\boldsymbol{v}_{2}^{2}$ & 0 & 0 & $(c+1) \boldsymbol{v}_{2}^{2}$ & $(c+1) \boldsymbol{v}_{2}^{2}$ \\
$\boldsymbol{v}_{2}^{3}$ & $-(c+1) \boldsymbol{v}_{2}^{1}$ & $-(c+1) \boldsymbol{v}_{2}^{2}$ & 0 & 0 \\
$\boldsymbol{v}_{2}^{4}$ & $-2(c+1) \boldsymbol{v}_{2}^{1}$ & $-(c+1) \boldsymbol{v}_{2}^{2}$ & 0 & 0 \\
\hline
\end{tabular}

$$
t_{R}=\sigma^{b} e^{\frac{a}{\log \sigma-\beta T}+(\alpha-b \beta) T} .
$$

Those formulations of the constitutive laws are compatible with the observed master curves. We can now proceed to the Lie analysis of these constitutive equations.

The Lie algebra of the creep model is computed following the methodology described by [5]. It contains 4 generators $v_{2}^{i}, i=1, \cdots, 4$ with prolongations given by:

$$
\begin{aligned}
& \operatorname{pr}^{(1)} \boldsymbol{v}_{2}^{1}=\sigma^{d} t^{-c} \frac{\partial}{\partial t}+c \dot{\varepsilon} \sigma^{d} t^{-c-1} \frac{\partial}{\partial \dot{\varepsilon}} \\
& \operatorname{pr}^{(1)} \boldsymbol{v}_{2}^{2}=\sigma^{d} \varepsilon^{1-v} \frac{\partial}{\partial \varepsilon}+(1-v) \sigma^{d} \varepsilon^{-v} \dot{\varepsilon} \frac{\partial}{\partial \dot{\varepsilon}} \\
& \operatorname{pr}^{(1)} \boldsymbol{v}_{2}^{3}=t \frac{\partial}{\partial t}+\left(\frac{c+1}{v}\right) \varepsilon \frac{\partial}{\partial \varepsilon}+\left(\frac{c+1-v}{v}\right) \dot{\varepsilon} \frac{\partial}{\partial \dot{\varepsilon}}, \\
& \operatorname{pr}^{(1)} \boldsymbol{v}_{2}^{4}=t \frac{\partial}{\partial t}-\left(\frac{c+1}{d}\right) \sigma \frac{\partial}{\partial \sigma}-\dot{\varepsilon} \frac{\partial}{\partial \dot{\varepsilon}}
\end{aligned}
$$

in which we recall that $v=\gamma+c \gamma+d \delta$. The commutation table of this Lie algebra is given in Table 2. In order to show the practical interest of this table, let us consider the particular combination of the calculated generators:

$$
\frac{v}{c+1}\left(\operatorname{pr}^{(1)} \boldsymbol{v}_{2}^{3}+\left(\frac{c+1-v}{v}\right) \operatorname{pr}^{(1)} \boldsymbol{v}_{2}^{4}\right)=t \frac{\partial}{\partial t}+\varepsilon \frac{\partial}{\partial \varepsilon}-\left(\frac{c+1-v}{d}\right) \sigma \frac{\partial}{\partial \sigma}
$$

which has no component with respect to $\dot{\varepsilon}$ and which is obviously a Lie symmetry of (3.31). The flow of this generator is given in logarithmic double axes by:

$$
\left\{\begin{aligned}
\log \bar{t} & =\mu+\log t \\
\log \bar{\sigma} & =-\left(\frac{c+1-v}{d}\right) \mu+\log \sigma \\
\log \bar{\varepsilon} & =\mu+\log \varepsilon .
\end{aligned}\right.
$$

This last set of relations is of great interest since it describes a master curve in the $\log \varepsilon$ vs. $\log t$ representation. More precisely, the curve $\log \bar{\varepsilon}$ vs. $\log \bar{t}$ obtained for a stress level $\bar{\sigma}$ is linked to the curve obtained for $\sigma$ by a translation of vector $(\mu, \mu)$, the shift being defined by $\mu=\frac{d}{c+1-v} \log (\sigma / \bar{\sigma})$.

The Lie algebra of the rupture model has two generators $\boldsymbol{v}_{1}^{i}, i=1,2$, given by:

$$
\begin{aligned}
& \boldsymbol{v}_{1}^{1}=t_{R}\left(\frac{b(\log \sigma-\beta T)^{2}-a}{\log \sigma-\beta T}\right) \frac{\partial}{\partial t_{R}}+\sigma(\log \sigma-\beta T) \frac{\partial}{\partial \sigma} \\
& \boldsymbol{v}_{1}^{2}=t_{R}\left(\frac{(\alpha-b \beta)(\log \sigma-\beta T)^{2}+a \beta}{\log \sigma-\beta T}\right) \frac{\partial}{\partial t_{R}}+(\log \sigma-\beta T) \frac{\partial}{\partial T}
\end{aligned}
$$


Table 3 Commutator table of the Lie algebra of Eq. (3.32)

\begin{tabular}{lll}
\hline & $\boldsymbol{v}_{1}^{1}$ & $\boldsymbol{v}_{1}^{2}$ \\
\hline $\boldsymbol{v}_{1}^{1}$ & 0 & $\beta \boldsymbol{v}_{1}^{1}+\boldsymbol{v}_{1}^{2}$ \\
$\boldsymbol{v}_{1}^{2}$ & $-\beta \boldsymbol{v}_{1}^{1}-\boldsymbol{v}_{1}^{2}$ & 0 \\
\hline
\end{tabular}

Table 4 Some creep laws encountered in the literature (see, e.g., Ref. [61])

\begin{tabular}{ll}
\hline References & Relationship \\
\hline$[71,72]$ & $\varepsilon=f_{1}(\sigma) f_{2}(t) f_{3}(T)$ \\
{$[73-76]$} & $\dot{\varepsilon}=f_{1}(\sigma) f_{2}(t) f_{3}(T)$ \\
{$[73,74,77,78]$} & $\dot{\varepsilon}=g_{1}(\sigma) g_{2}\left(\varepsilon_{c}\right) g_{3}(T)$ \\
{$[74]$} & $E(t) \Phi[\varepsilon(t)]=\sigma(t)+\int_{0}^{t} K(t, \tau) \sigma(\tau) d \tau$ \\
\hline
\end{tabular}

and its commutator table is given in Table 3. If we denote by $\phi^{1, t_{R}}$ and $\phi^{2, t_{R}}$ the component in $t_{R}$ of $v_{1}^{1}$ and $v_{1}^{2}$ respectively, then the following local combination:

$$
\begin{aligned}
\boldsymbol{v}= & \frac{\phi^{2, t_{R}} \boldsymbol{v}_{1}^{1}-\phi^{1, t_{R}} \boldsymbol{v}_{1}^{2}}{t_{R}}=\sigma\left[a \beta+(\alpha-b \beta)(\log \sigma-\beta T)^{2}\right] \frac{\partial}{\partial \sigma} \\
& +\left(a-b(\log \sigma-\beta T)^{2}\right) \frac{\partial}{\partial T}
\end{aligned}
$$

is a symmetry of Eq. (3.32) having no component in $t_{R}$ and whose components in $\sigma$ and $T$ do not depend on $t_{R}$. Consequently, $v$ has the form of Eq. (2.4), and the flow of this vector field is given by the solution of the system of differential equations (2.6):

$$
\begin{aligned}
& \frac{d \bar{\sigma}}{d \mu}=\bar{\sigma}\left[a \beta+(\alpha-b \beta)(\log \bar{\sigma}-\beta \bar{T})^{2}\right] \\
& \frac{d \bar{T}}{d \mu}=a-b(\log \bar{\sigma}-\beta \bar{T})^{2}
\end{aligned}
$$

with the initial conditions

$$
\bar{\sigma}(0)=\sigma, \quad \bar{T}(0)=T
$$

and the equality $\bar{t}_{R}=t_{R}$. Consequently, the numerical solution of (3.42) and (3.43) provides a parametric representation $(T(\mu), \sigma(\mu))$ of the "iso- $t_{R}$ " curves in the $(T, \sigma)$ plane, see Fig. 13. This kind of theoretical chart is of high practical interest, as it allows to extrapolate the rupture time of a specimen at any stress level or any temperature, provided that the rupture time is known for a given value of $\sigma$ and $T$.

Summarizing this section, material data have been given the structure of experimental Lie groups, using as a subsequent step the Lie group structure to construct a material constitutive law in terms of initially unknonw constitutive functions. The Lie symmetries can accordingly be considered as an interpolation method enabling to link in a continuous manner experimental data, but relying on a well-chosen limited set of experimental measurements.

\section{Invariance relations and master responses associated to a rheological model}

The formulation of the constitutive model including a set of parameters is here followed by the symmetry analysis, leading to invariance relations and master responses inherent to the model. In the context of rheology, this approach proves quite promising for dissipative material with a complex nonlinear constitutive law, including the state laws satisfied by the observable variables and the kinetics of internal variables traducing the evolution 


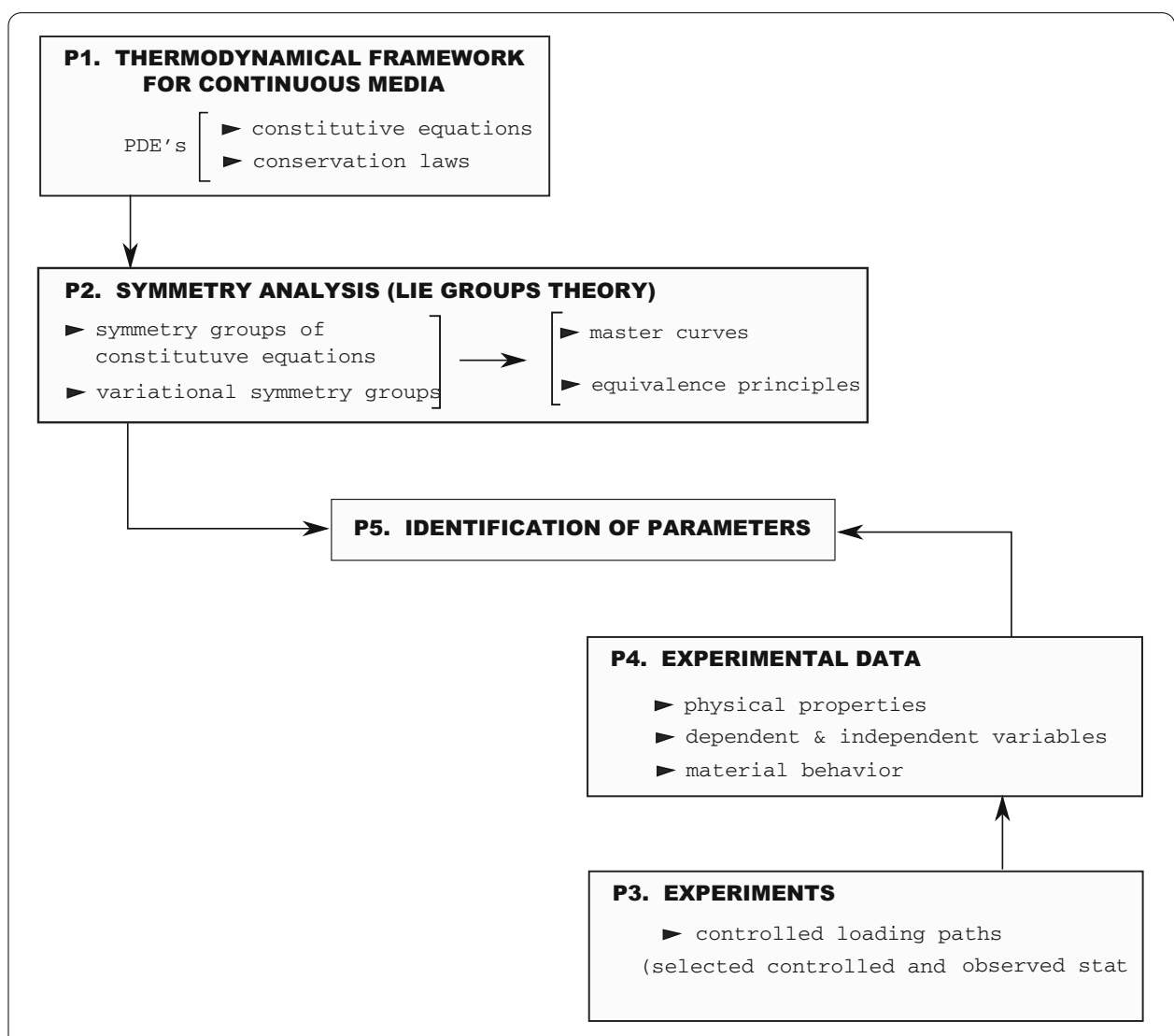

Fig. 1 Construction and predictions of constitutive models from data and symmetry methods (see Ref. [41])

laws of the internal variables. The invariants associated to those symmetries can then be calculated and used to synthesize the material's response into master responses reflecting the material's response when the control parameters change. The proposed algorithm reflecting the entire methodology is summarized in the diagram of Fig. 1. The formulation of rheological models can be guided by the framework of the thermodynamics of irreversible processes, as explained in the sequel.

\section{Construction of the constitutive laws in the framework of TIP}

In mechanics or rheology, constitutive laws for dissipative materials are generally written under the umbrella of the thermodynamics of irreversible processes (TIP) [42], relying on the energy density to describe the energetic part of the constitutive law and the dissipation potential for its dissipative part. Relying on Callen's axiomatic, [43], and a generalization of De Donder's thermodynamics, let the total internal energy $E(\boldsymbol{y}, \boldsymbol{z})$ characterizing the medium depend on a set of extensive variables $y=\left(S, V \varepsilon, N_{k} \ldots\right)$, where $S, V \varepsilon$, and $N_{k}$ are respectively the entropy, the volume weighted deformation, the number of moles of the $k^{\text {th }}$ species, further identified to a set of internal variables $z$ describing the irreversible evolution of the microstructure (such as plasticity, viscoplasticity, and damage). The thermodynamical system is a finite volume $V$ with boundary $\partial V$ of a solid continuum body. The extensity property of $E$ expresses as

$$
E(\lambda \boldsymbol{y}, \lambda \boldsymbol{z})=\lambda E(\boldsymbol{y}, \boldsymbol{z})
$$


Taking the derivative of previous equation with respect to $\lambda$ at $\lambda=1$, the Euler relation is obtained in the form of the Gibbs relation

$$
E(\boldsymbol{y}, \boldsymbol{z})=\boldsymbol{Y}(\boldsymbol{y}, \boldsymbol{z}) \cdot \boldsymbol{y}-\boldsymbol{A}(\boldsymbol{y}, \boldsymbol{z}) \cdot \boldsymbol{z}
$$

satisfied by the internal energy $E(\boldsymbol{y}, \boldsymbol{z})$, with $\boldsymbol{Y}=E_{, y}$ the intensive variables (vector) and $\boldsymbol{A}=-E_{, z}$ the generalized non equilibrium forces respectively. The intensive variables are dual of the extensive ones in a thermodynamical sense. For example, the second order Cauchy stress tensor $\sigma$, is conjugated to the second order strain tensor $\varepsilon$. The extensive variables are called control variables, since they are the arguments of an appropriate thermodynamical potential. Accounting for the Gibbs relation:

$$
\frac{d E}{d t}(\boldsymbol{y}, \boldsymbol{z})=\boldsymbol{Y}(\boldsymbol{y}, \boldsymbol{z}) \cdot \frac{d \boldsymbol{y}}{d t}-A(\boldsymbol{y}, \boldsymbol{z}) \cdot \frac{d z}{d t},
$$

the Gibbs-Duhem relation results from the differentiation of Eq. (4.2), viz

$$
y \cdot \frac{d Y}{d t}-z \cdot \frac{d A}{d t}=0
$$

The constitutive law is specified in rate form from the total time derivation of the intensive variables $\boldsymbol{Y}$ and $\boldsymbol{A}$ as

$$
\begin{aligned}
\dot{Y}(y, z) & =a^{u}(y, z) \cdot \dot{y}+b(y, z) \cdot \dot{z} \\
-\dot{A}(y, z) & =b^{T}(y, z) \cdot \dot{y}+g(y, z) \cdot \dot{z}
\end{aligned}
$$

with $\boldsymbol{a}^{\boldsymbol{u}}(\boldsymbol{y}, \boldsymbol{z})=E_{, y y}$ the Tisza matrix, $\boldsymbol{b}(\boldsymbol{y}, \boldsymbol{z})=E_{, z y}, \boldsymbol{g}(\boldsymbol{y}, \boldsymbol{z})=E_{, z z}$ the coupling and the dissipation matrices respectively, [44].

In the more general setting of the theory of irreversible process (abbreviated T.I.P.) or the thermodynamics with internal variables (abbreviated T.I.V.), the kinetic laws of the internal variables are written based on the setting up of the dissipation potential $D$, a positive and homogeneous function of degree $n$ in the rate of the internal variables, [42], allowing to express the thermodynamic forces or affinities reflecting all dissipative variables $\boldsymbol{A}$ versus the dual internal variable $\boldsymbol{z}$ as

$$
\boldsymbol{A}(\boldsymbol{z})=\frac{\partial D}{\partial \dot{z}} .
$$

Using previous relation results in a dissipation expressing as

$$
\Phi=\boldsymbol{A} \dot{\boldsymbol{z}}=\frac{\partial D(\boldsymbol{z})}{\partial \dot{z}}=n D \geq 0
$$

due to Euler's identity for homogeneous functions of degree $n$ (in classical T.I.P., $D$ is taken as homogeneous of degree two). The previous relation can be inverted to give the rate of the internal variable versus the affinity, using the Legendre-Fenchel transform, involving the pseudo-potential of dissipation, defined as

$$
D^{*}(\boldsymbol{A})=\sup _{\boldsymbol{A}}(\boldsymbol{A} \dot{\boldsymbol{z}}-D(\dot{\boldsymbol{z}}))
$$

where $\boldsymbol{A}$ is restricted to a convex set $K$, [42]. When $D^{*}(\boldsymbol{A})$ is differentiable, the evolution law of the internal variable $z$ is given by

$$
\dot{\boldsymbol{z}}=\frac{\partial D^{*}(\boldsymbol{A})}{\partial \boldsymbol{A}} \text {. }
$$

Choosing as a specific model a quadratic and convex pseudo potential of dissipation $D^{*}$, one obtains the following set of independent non linear kinetic relations (for a number of $N$ processes)

$$
\dot{z}_{k}=-\frac{z_{k}-z_{k}^{r}}{\tau_{k}}, \quad k=1 . . N
$$


considering a spectral decomposition of dissipative phenomena modelled by internal variables $\dot{z}_{k}$. The variables $z_{k}^{r}$ represent the value of the internal variable $z$ at its relaxed equilibrium state, for the dissipation mode $k$, defined as the state for which the thermodynamic affinity $A^{r}$ vanishes. The distribution of the relaxation times therein $\tau_{k}$ is described from Prigogine's fluctuation theorem, the contribution of each mode being proportional to the square root of the corresponding relaxation time [44].

A Lagrangian formulation of the previous dissipative constitutive laws is further constructed by the homotopy formula from the self-adjoint system of PDE's (4.5), accounting for the Gibbs relation Gibbs-Duhem relations (4.4), see [45,46]:

$$
L=E_{, y} \cdot \dot{\boldsymbol{y}}+E_{, z} \cdot \dot{\boldsymbol{z}}=\frac{d E(\boldsymbol{y}, \boldsymbol{z})}{d t} .
$$

This thermodynamic Lagrangian incorporates the thermodynamic information related to the material, in terms of relations between the extensive control variables and the dual intensive thermodynamic forces, where the generalized coordinates $\{\boldsymbol{y}, \boldsymbol{z}, \dot{\boldsymbol{y}}, \dot{\boldsymbol{z}}\}$ correspond to the Lagrange variables of the considered spatially uniform system. The set of internal variables completes the set of control variables, ensuring the self-adjointness of the constitutive equations [45]. The kinetic information, viz the set of equations (4.10) is then incorporated into the previous thermodynamic Lagrangian via Lagrange multipliers $\lambda=\left\{\lambda_{k}, k=1 . . N\right\}$, hence the augmented Lagrangian, $L_{\text {aug }}$, accounting for both the thermodynamic and the kinetic information inherent to the material's constitutive law, writes

$$
l_{\text {aug }}=\dot{e}+\sum_{k=1}^{N} \lambda_{k}\left(\dot{z}_{k}+\frac{z_{k}-z_{k}^{r}}{\tau_{k}}\right),
$$

where the internal energy $E$ has been replaced by the internal energy density $e$, which also satisfies the Maxwell conditions. The Lagrangian density $l_{\text {aug }}$ in Eq. (4.12) is the volumetric density of a Lagrangian $L_{\text {aug }}$, such that

$$
L_{\text {aug }}=\int_{V} l_{\text {aug }} d V
$$

The Lagrange multipliers in $l_{\text {aug }}$ resemble thermodynamic affinities dual to the rate of internal variables, hence the kinetic part of the total Lagrangian thereabove resembles a dissipation potential. Observe that the incorporation of the kinetic laws Eq. (4.10) clearly breaks the symmetry (discrete symmetry) under time reversal $t \rightarrow-t$, since the new Lagrangian contains an irreversible information. The set of Lagrange equations obtained from the stationarity condition of the action functional built from $l_{\text {aug }}$ is completely equivalent to the constitutive equations (4.5) and the kinetic laws (4.10). The kinetic evolution equations Eq. (4.9) have symmetry properties depending on the specific form taken by the relaxation time therein.

\section{Symmetry analysis of the augmented Lagrangian}

Relying on the formulated least action principle of the dissipative constitutive laws, we next exploit the associated variational symmetries to determine invariance properties of the set of constitutive equations (thermodynamic and kinetic informations), relying on the methodology presented in [45-48]. We focus in the sequel (but without lost of generality) on the specific energy $e\left(\varepsilon, s, z_{k}\right)$, depending on the strain $\varepsilon$, the specific entropy $s$, and some specific internal variables $z_{k}, k=1 . . N$, accounting for internal dissipative phenomena (related to the microstructure). 
The search for the variational symmetries of the Jacobi action built from the augmented Lagrangian amounts to find the infinitesimal generators on the (first order) jet space sustained by the control variables $\left\{t, \varepsilon, s, z_{k}\right\}$. As a concrete illustration, and without loss of generality, considering the time $t$ as the sole independent variable and as the dependent variables the vector $\boldsymbol{y}=\varepsilon, s, z_{k}$, a generator of a symmetry group expresses as

$$
\boldsymbol{v}_{\text {cont }}=\xi \frac{\partial}{\partial t}+\phi^{\varepsilon} \frac{\partial}{\partial \varepsilon}+\phi^{s} \frac{\partial}{\partial s}+\phi^{z_{k}} \frac{\partial}{\partial z_{k}}
$$

with the variations of the dependent variables given by

$$
\delta \varepsilon=\mu \phi^{\varepsilon} ; \delta s=\mu \phi^{s} ; \delta z_{k}=\mu \phi^{z_{k}},
$$

with $\mu$ the group parameter, [5]. In Eq. (4.14), the subscript "cont" means that only components with respect to control variables are at first introduced. The variations given in Eq. (4.15) are responsible for the variations of intensive observable variables $\delta \sigma, \delta T$, and $\delta A_{k}$, obtained from the second partial derivatives of the potential $e$. The symmetry group acting on the set of variables $\left\{t, \varepsilon, s, z_{k}\right\}$ is automatically extended to the enlarged set of variables $\left\{t, \varepsilon, s, z_{k}, \sigma, T, A_{k}\right\}$ : thereby, the total vector field, generator of the symmetries of the constitutive laws, is decomposed into the sum of the "control" vector field and the "observable" vector field:

$$
\boldsymbol{v}=\boldsymbol{v}_{\text {cont }}+\boldsymbol{v}_{\text {obs }} \text { with } \quad \boldsymbol{v}_{o b s}=\phi^{\sigma} \frac{\partial}{\partial \sigma}+\phi^{T} \frac{\partial}{\partial T}+\phi^{A_{k}} \frac{\partial}{\partial A_{k}} .
$$

The components of the intensive variables of the observable and internal vector fields are given by the structure of the constitutive law (Eq. 4.5) for the elementary representative volume element (RVE):

$$
\begin{aligned}
\phi^{\sigma} & =e_{, \varepsilon \varepsilon} \phi^{\varepsilon}+e_{, s \varepsilon} \phi^{s}+e_{, z_{k} \varepsilon} \phi^{z_{k}} \\
\phi^{T} & =e_{, \varepsilon s} \phi^{\varepsilon}+e_{, s s} \phi^{s}+e_{z_{k} s} \phi^{z_{k}} \\
\phi^{A_{i}} & =e_{, \varepsilon z_{i}} \phi^{\varepsilon}+e_{, s z_{i}} \phi^{s}+e_{z_{k} z_{i}} \phi^{z_{k}} .
\end{aligned}
$$

The components of the vector field $\boldsymbol{v}_{o b s}$ are thus completely determined by those of the vector field $\boldsymbol{v}_{\text {cont }}$. Let return now to a general situation, and decompose the Lagrangian of Eq. (4.12) into a thermodynamic and a kinetic contribution, as

$$
l_{\text {aug }}=l_{\text {thermo }}+l_{\text {kine }}
$$

with

$$
l_{\text {thermo }}=\dot{e} \quad \text { and } \quad l_{\text {kine }}=\sum_{k=1}^{N} \lambda_{k}\left(\dot{z}_{k}+\frac{z_{k}-z_{k}^{r}}{\tau_{k}}\right) .
$$

As shown previously, the invariance condition associated with the sole contribution $l_{\text {thermo }}$ is automatically verified, see also [45]. The satisfaction of the Euler-Lagrange equations for $\dot{e}$ as a Lagrangian is fully equivalent to the Maxwell conditions for the second order partial derivatives of the potential function $e$. Thus, the invariance condition of the action integral simplifies to the differential condition involving the sole kinetic information (and kinetic Lagrangian)

$$
\mathrm{pr}^{(1)} \boldsymbol{v} l_{\text {kine }}+l_{\text {kine }} \operatorname{Div} \xi=0 \text {, }
$$

accounting for the relation $\operatorname{Div} \xi=D_{t} \xi=\dot{\xi}$. 
The connection between the variational symmetry condition and the local symmetry of the field equations is next established. If $\boldsymbol{v}$ is a variational symmetry for some functional $S=\int_{t} \int_{V} l d V d t$, it is also a symmetry of the corresponding Lagrange equations:

$$
\operatorname{pr}^{(1)} \boldsymbol{v} l+l \operatorname{Div} \xi=0 \Rightarrow \operatorname{pr}^{(1)} \boldsymbol{v}(\boldsymbol{E}(l))=0,
$$

but the converse is generally not true: the set of all variational symmetries denoted by $G_{S}$ is always included into the set of local symmetries, $G_{\Delta}$, i.e. $G_{S} \subset G_{\Delta}$. In the present case, we consider only variational symmetries along the optimal path where the constitutive laws are satisfied. Accounting for the expression of $l_{\text {kine }}$, viz

$$
l_{\text {kine }}=\sum_{k=1}^{N} \lambda_{k}\left(\dot{z}_{k}+\frac{z_{k}-z_{k}^{r}}{\tau_{k}}\right),
$$

the condition $l_{\text {kine }}=0$ is satisfied along the optimal path, hence the variational symmetry condition resumes to

$$
\mathrm{pr}^{(1)} \boldsymbol{v} l_{\text {kine }}=0 \text { whenever } l_{\text {kine }}=0 \text {. }
$$

Thanks to the linearity of the prolongation and doing a spectral decomposition of the dissipative mechanisms, the evolution of the internal variables are mutually independent, hence the symmetry conditions (4.25) may be decoupled, resulting in the new set of $N$ independent symmetry conditions:

$$
\operatorname{pr}^{(1)} \boldsymbol{v}\left(\dot{z}_{k}+\frac{z_{k}-z_{k}^{r}}{\tau_{k}}\right)=0 \text { whenever } \dot{z}_{k}+\frac{z_{k}-z_{k}^{r}}{\tau_{k}}=0,
$$

which is equivalent to the local symmetry condition.

The same condition holds for the more general evolution equation (4.9), thus allowing to extend the symmetry analysis to more complex dissipative phenomena. A classification of symmetries according to the form of the pseudo-potential of dissipation can also be done. Previous considerations entail that variational symmetries along the optimal path are fully equivalent to local symmetries directly computed from the constitutive laws.

The symmetries of the formulated augmented lagrangian formulation are those of the pseudo-potential of dissipation $D^{*}(\boldsymbol{A})$. The infinitesimal generator of the general invariance condition (4.25) is also split into the sum of a control and an observable vector field, with the components of the last contribution completely expressed from the components of the control vector; this implies searching for symmetries in a subset of the total jet space, since the state laws for the observable variables are automatically satisfied.

The presented general thermodynamically based material framework originates in pioneering works [49-52], and it covers a broad spectrum of rheological models in viscoelasticity, viscoplasticity, plasticity and also continuum damage mechanics.

In the next section, the invariance properties of the constitutive equations are exploited to set up a predictive methodology allowing to condense the materials response into equivalence principles. This has consequences on the experimental side, since the later enable to define equivalent experimental set up allowing a gain of time by selecting an optimal set of control variables (e.g. temperature, strain rate).

\section{Invariance properties of the constitutive equations}

Considering for instance a general internal variable formulation of inelasticity for generalized standard materials, the symmetries of the constutive laws are implicitly reflected 
by the form taken by the thermodynamic potential (or the pseudo potential of dissipation introduced in Eq. (4.8)) and the dissipation function. The search for the Lie groups of constitutive laws is further used as a systematic tool for the construction of the so-called master curves, that condense the information related to the behavior of a material under varying experimental conditions. The experimental conditions are defined by the values taken by a set of control parameters such as temperature, strain rate. Hence, the knowledge of symmetry groups allows a prediction regarding the modification of the material's response when these parameters vary. Thus, starting from a known set of constitutive equations, i.e. a known expression for $e, z_{k}^{r}$ and $\tau_{k}$, it is a priori possible to compute some symmetries of the behavior by applying the symmetry condition (4.26). Due to the equivalence of the local and variational symmetry conditions, a conservation law may be obtained using Noether's theorem, $\operatorname{viz} \operatorname{Div} \boldsymbol{P}=0$, with

$$
P_{i}=\sum_{k=1}^{q} \sum_{j=1}^{4} \xi_{j} u_{k, j} \frac{\partial l_{k i n e}}{\partial u_{k, i}}-\sum_{j=1}^{q} \phi_{j} \frac{\partial l_{k i n e}}{\partial u_{j, i}}-\xi_{i} l_{k i n e},
$$

see e.g. [5], with $l_{\text {kine }}$ the kinetic Lagrangian density, defined in the general thermodynamic setting exposed in the previous section as

$$
l_{\text {kine }}=\sum_{k} \lambda_{k}\left(\dot{z}_{k}-\frac{\partial D^{*}(\boldsymbol{A})}{\partial A_{k}}\right) \equiv 0 .
$$

Recall that the dependent variables arguments of the kinetic Lagrangian, viz the variables $u_{k}$, are internal variables. For a uniform elementary representative volume element, only the time derivative appears in previous conservation law expression; BVPs accounting for the spatial variation of the fields over spatial domains will be considered later on in this contribution.

\section{Application: construction of the master curve of a dry polyamid (PA66) and time temperature equivalence}

The invariants build from the Lie symmetry analysis of the constitutive law will further be translated into master curves, as explained below. The constitutive model set up presently for the purpose of uniaxial tests involves the strain $\epsilon$ as control variable, and the stress $\sigma$ as the corresponding observable variable. The temperature $T$, dual of the entropy $s$, plays the role of a parameter. The internal variables $z_{k}$ and the thermodynamic affinities $A_{k}$ are not controlled; their values at the relaxed state are governed by the strain history. The exploitation of the general constitutive Eq. (4.5) and (4.10) delivers the following state law (see [53]):

$$
\left\{\begin{array}{l}
\dot{\sigma}-E_{u} \dot{\varepsilon}+\sum_{k=1}^{n} b_{k}^{1} \frac{z_{k}-z_{k}^{r}}{\tau_{k}}=0 \\
-\dot{s}+\alpha_{u} E_{u} \dot{\varepsilon}+\sum_{k=1}^{n} b_{k}^{2} \frac{z_{k}-z_{k}^{r}}{\tau_{k}}=0 \\
-\dot{A}_{i}-b_{i}^{1} \dot{\varepsilon}+\sum_{k=1}^{n} g_{i k} \frac{z_{k}-z_{k}^{r}}{\tau_{k}}=0 \quad i=1 . . n
\end{array}\right.
$$

with $E_{u}$ the constant instantaneous Young's modulus, $b_{k}^{1}, \alpha_{u}$ (dilatation coefficients), $b_{k}^{2}$, and $g_{i k}$ being defined from the second order partial derivatives of the Helmholtz free energy, see [54]. The simplest model for the evolution of the internal variables is

$$
z_{k}^{r}=c_{k} \varepsilon
$$


If one starts from a particular expression of the kinetic laws, involving a temperature dependence of the relaxation times of Arrhenius kind, the following kinetic model may be written

$$
\dot{z}_{k}+\frac{z_{k}-c_{k} \varepsilon}{\frac{h}{k T} \exp \left(\frac{\Delta H-T \Delta S_{k}}{R T}\right)}=0,
$$

where $h$ is the Planck constant, $k$ the Boltzmann constant, $R$ the gas constant, $c_{k}, \Delta H$, $\Delta S_{k}$ some material constants. The required symmetry condition expresses accordingly

$$
\operatorname{pr}^{(1)} \boldsymbol{v}\left(\dot{z}_{k}+\frac{z_{k}-c_{k} \varepsilon}{\frac{h}{k T} \exp \left(\frac{\Delta H-T \Delta S_{k}}{R T}\right)}\right)=0 .
$$

The particular solution is obtained

$$
\boldsymbol{v}_{0}=\xi \frac{\partial}{\partial t}+\phi^{T} \frac{\partial}{\partial T}+\phi^{\sigma} \frac{\partial}{\partial \sigma}+\phi^{s} \frac{\partial}{\partial s}+\phi^{A_{k}} \frac{\partial}{\partial A_{k}}
$$

with:

$$
\begin{gathered}
\xi=t ; \quad \phi^{T}=-\frac{R T^{2}}{R T+\Delta H} ; \phi^{\sigma}=-\alpha_{u} E_{u} \phi^{T} ; \\
\phi^{A_{k}}=-b_{k}^{2} ; \phi^{T} ; \phi^{\varepsilon}=0 ; \quad \phi^{z_{k}}=0 .
\end{gathered}
$$

This solution is further interpreted as a mathematical formulation of the so-called time-temperature equivalence principle for polymers. The integration of the induced first order differential system leads to a one-parameter group of transformations, where the temperature is transformed as

$$
\begin{aligned}
\bar{T}= & \exp \left(L_{W}\left(\frac{\Delta H}{R} \exp \left(\frac{\mu T-T \ln \left(T^{*}\right)+\frac{\Delta H}{R}}{T}\right)\right)\right. \\
& \left.-\mu+\ln T^{*}-\frac{\Delta H}{R T}\right)
\end{aligned}
$$

with $L_{W}(x)$ the Lambert function, and $T^{*}=T / T_{0}$ with $T_{0}=1 \mathrm{~K}$. The theoretical shift factor is obtained by inverting the previous implicit relation:

$$
\frac{\mu(T, \bar{T})}{\ln 10}=\frac{\Delta H(T-\bar{T})}{R T \bar{T} \ln 10}+\frac{1}{\ln 10} \ln \frac{T}{\bar{T}} .
$$

This explicit expression of the group parameter $\mu(T, \bar{T})$ highlights an invariance property satisfied by the secant modulus $E_{S}(t, T)$, defined as the ratio of stress to strain:

$$
E_{s}(t, T)=\frac{\sigma(t, T)}{\varepsilon(t, T)}=\frac{\bar{\sigma}(\bar{t}, \bar{T})}{\bar{\varepsilon}(\bar{t}, \bar{T})}=E_{s}(\bar{t}, \bar{T})=E_{s}\left(e^{\mu} t, \bar{T}\right)
$$

leading to the logarithmic relation between times:

$$
\log \bar{t}=\log t+\frac{\mu}{\ln 10}
$$

This allows rewriting Eq. (4.37) under the form:

$$
E_{s}(\log t, T)=E_{s}\left(\log t+\frac{\mu}{\ln 10}, \bar{T}\right) \text {. }
$$

This relation links the two secant moduli $E_{s}(\log \bar{t}, \bar{T})$ and $E_{s}(\log t, T)$ obtained at $\bar{T}$ and $T$ by a translation by $\frac{\mu}{\ln 10}$ on the time logarithmic scale, given explicitly in Eq. (4.36). The obtained invariance property is a theoretical formulation of the time-temperature equivalence principle. The latter was successfully confirmed by experimental data on 


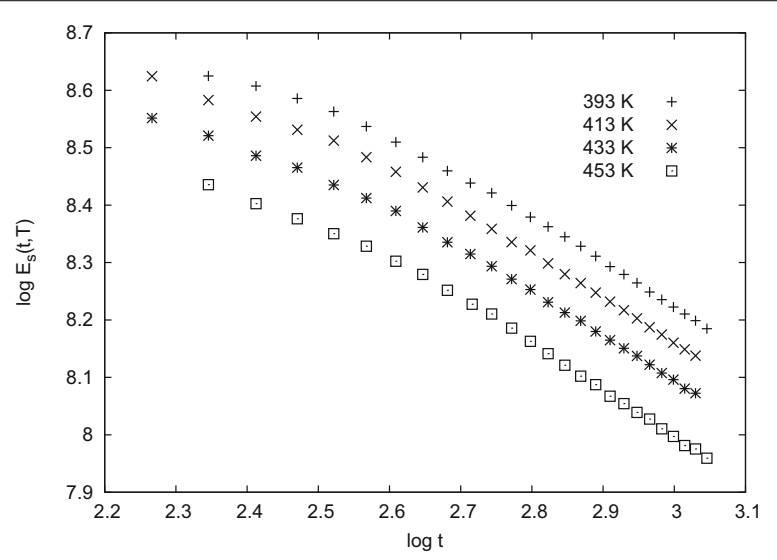

Fig. 2 Experimental secant modulus $E_{s}(t, T)$ vs time $t$ (log-log representation) for PA66 at different temperatures, see Ref. [54]

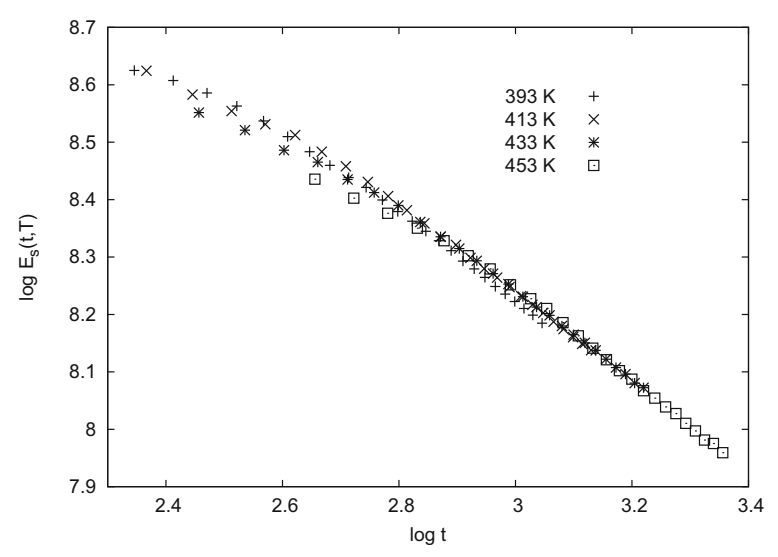

Fig. 3 Master curve obtained at 393K by translation of curves in Fig. 2 along the time axis, see Ref. [61]

various materials, and fitted with the empirical Williams-Landel-Ferry (WLF) expression (see e.g. [55-57]) or the Kohlrausch relation ([58-60]).

As an illustration of the time-temperature equivalence principle, and referring to the experimental validation of the predicted shift factor (DM-P5), let consider the data summarized in Fig. 2. The evolution of the secant modulus $E_{s}(t, T)$ for isothermal tests on a polymer (polyamid 66, PA66) is plotted at different constant temperatures and for a given strain rate $\left(\dot{\varepsilon}=1.8 \times 10^{-4} \mathrm{~s}^{-1}\right)$. Every curve is parameterized by the temperature $\bar{T}$ (in the range $413 \mathrm{~K}-453 \mathrm{~K}$ ) and can be translated along the $\log t$ axis with a horizontal shift factor $a_{\bar{T} \rightarrow T}$ to coincide with a unique curve. This unique curve is called "master curve" at the reference temperature $T=393 K$, see Fig. 3 .

The notion of equivalence transformation is exemplified below for the case of nonlinear wave propagation in fiber-reinforced materials, showing that the initial model including several parameters can be reduced to a model with no arbitrary parameters. 


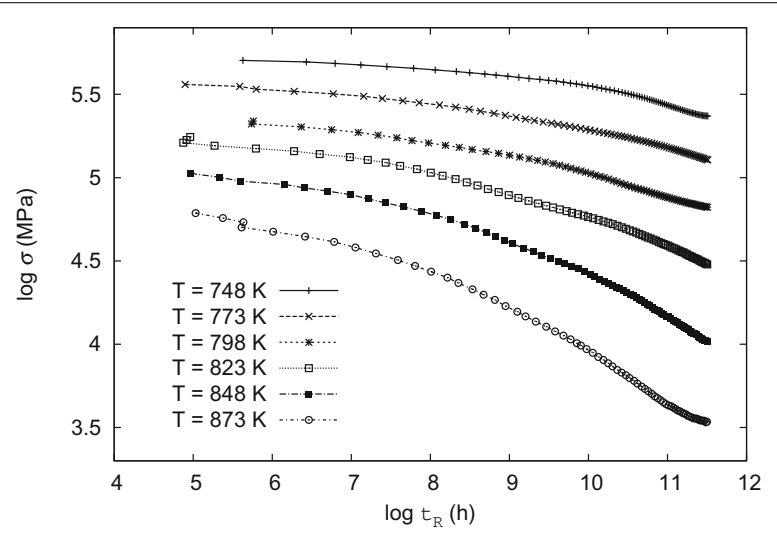

Fig. 4 Experimental stress vs. rupture at different temperatures, see Ref. [61]

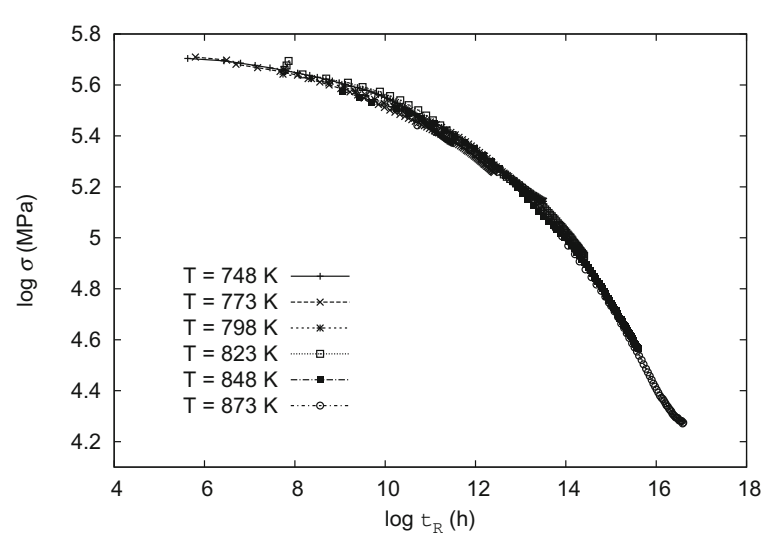

Fig. 5 Experimental master curve at the reference temperature $\bar{T}=748 \mathrm{~K}$ obtained by shifting the curves of Fig. 4 along the $\log t_{R}$ and $\log \sigma$ axis (from Ref. [61])

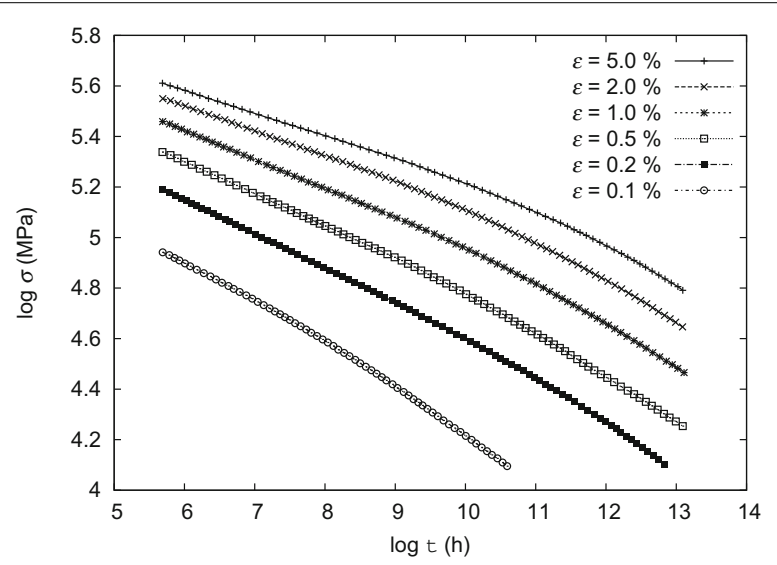

Fig. 6 Experimental stress vs. time responses at different strain levels in the logarithmic plane $(T=773 \mathrm{~K})$ (from Ref. [61]) 


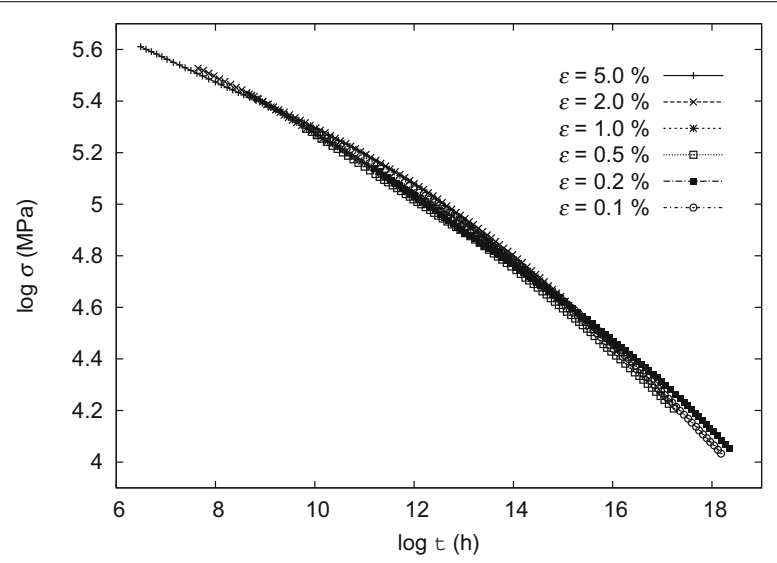

Fig. 7 Master curve obtained at the reference strain $\bar{\varepsilon}=5.0 \%$ by shifting the responses of Fig. 6 along the $\log t$ and $\log \sigma$ axis (from Ref. [61])

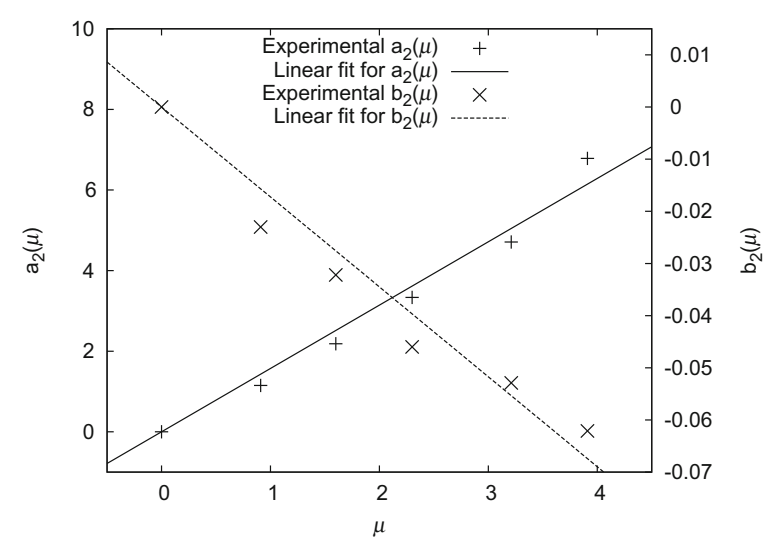

Fig. 8 Experimental curve and linear fit for $a_{2}(\mu)$ and $b_{2}(\mu)$ (from Ref. [61])

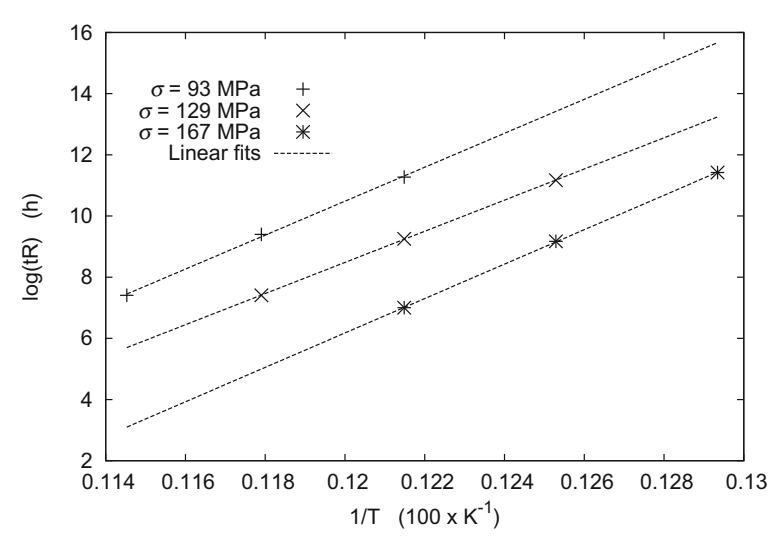

Fig. 9 Experimental responses $\log \left(t_{R}\right)$ vs. 1/T at different stress levels (Ref. [61]) 


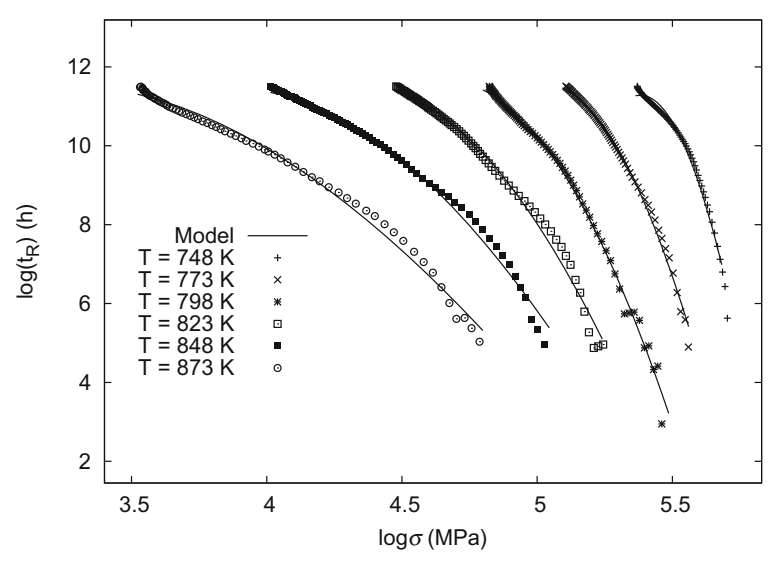

Fig. 10 Validation of Eq. (3.25) (from Ref. [61])

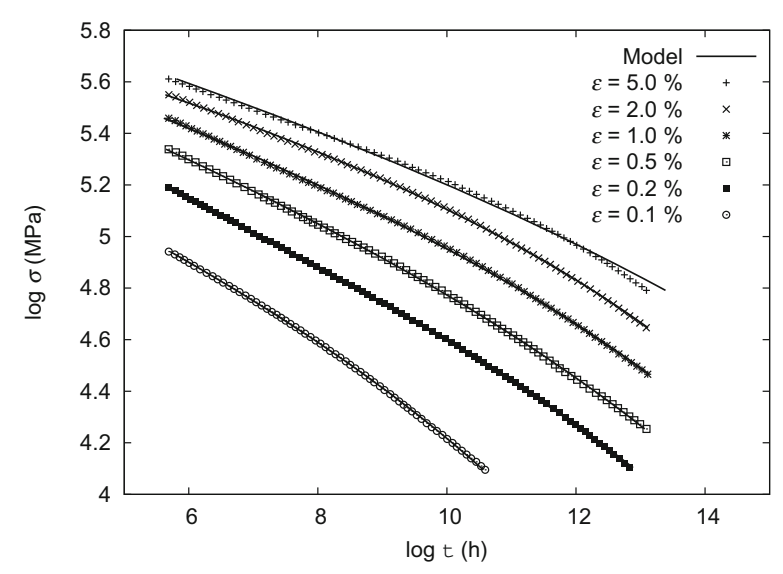

Fig. 11 Validation of Eq. (3.30) at $T=773 \mathrm{~K}$ (from Ref. [61])

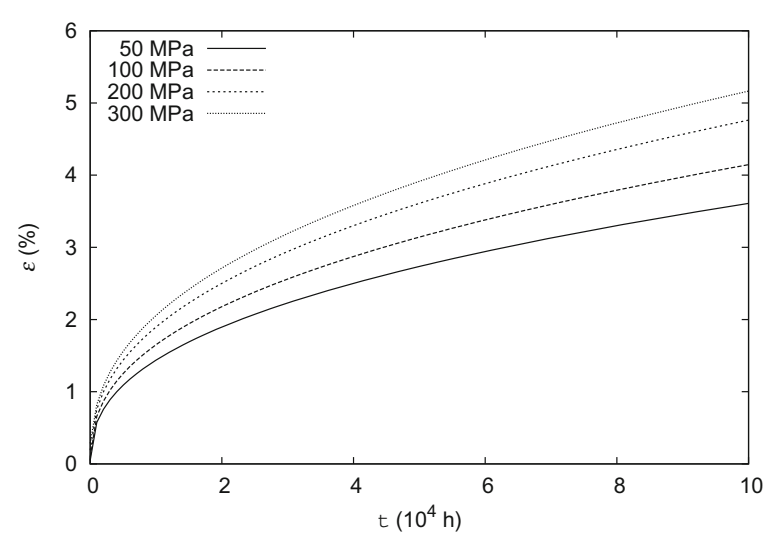

Fig. 12 Creep curves $\varepsilon(t)$ at different stress levels as predicted by the present model (from Ref. [61]) 


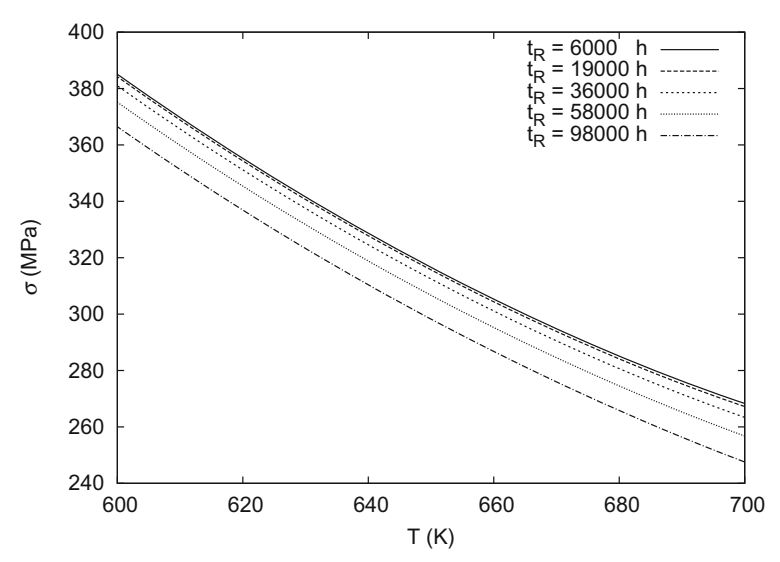

Fig. 13 Predicted "iso- $t_{R}$ " curves given by the numerical solution of Eqs. (3.42) and (3.43) (see Ref. [61])

\section{Nonlinear anti-plane shear wave models for fiber-reinforced materials: equivalence transformations and constitutive model simplification}

Multiple mechanical models of elastic structures such as biological and cell membranes, textiles, and rubbers, are modeled using the framework of incompressible hyperelasticity, which may include anisotropy effects such as a presence of elastic fibers [62]. In a solid body in three dimensions, the actual position $\boldsymbol{x}=\left(x_{1}, x_{2}, x_{3}\right)$ of a material point depends on its Lagrange coordinates $X=\left(X_{1}, X_{2}, X_{3}\right)$ and time $t$ :

$$
\boldsymbol{x}=\boldsymbol{\phi}(\boldsymbol{X}, t) .
$$

The deformation gradient is given by

$$
\boldsymbol{F}(\boldsymbol{X}, t)=\nabla \boldsymbol{\phi}, \quad F_{i j}=\frac{\partial \phi_{i}}{\partial X_{j}} ;
$$

for the incompressible case, the Jacobian $J=\operatorname{det} \mathbf{F}=1$. The full system of equations of motion of an incompressible hyperelastic material in three dimensions is given by the Newton's second law (momentum balance equation) and the incompressibility condition:

$$
\rho_{0} \boldsymbol{x}_{t t}=\operatorname{div}_{(\boldsymbol{X})} \mathbf{P}+\rho_{0} \mathbf{R}, \quad J=\operatorname{det} \mathbf{F}=1 .
$$

In (5.2), $\rho_{0}$ is the Lagrangian mass density, $\mathbf{P}$ is the first Piola-Kirchhoff tensor computed from the strain energy density $W$, and $\boldsymbol{R}=\boldsymbol{R}(\boldsymbol{X}, t)$ is the total body force per unit mass. The arbitrary element present in this class of models is the functional form of the strain energy density function $W$ :

$$
W=W_{\text {iso }}\left(I_{1}, I_{2}\right)+W_{\text {aniso }}\left(I_{4}, \ldots, I_{9}\right),
$$

where $W_{i s o}$ and $W_{\text {aniso }}$ are isotropic and anisotropic contributions, respectively; $I_{1}, I_{2}$ are invariants of the right Cauchy-Green deformation tensor $\mathbf{C}=\mathbf{F}^{T} \mathbf{F}$, and $I_{4}, \ldots, I_{9}$ are pseudo-invariants involving fiber direction vectors (for details, see, e.g., [62,63], and references therein). A wide class of materials is described using Mooney-Rivlin isotropic energy part and the standard reinforcement anisotropic model [63]:

$$
W=a\left(I_{1}-3\right)+b\left(I_{2}-3\right)+q\left(I_{4}-1\right)^{2},
$$

where $a, b, q>0$ are constant material parameters. 
In [63], it has been shown that for a shear wave propagating in $X_{1}$-direction, i.e., with finite displacements $G\left(X_{1}, t\right)$ arising from the incompressibility-compatible ansatz

$$
\boldsymbol{x}=\left[\begin{array}{c}
X_{1} \\
X_{2} \\
X_{3}+G\left(X_{1}, t\right)
\end{array}\right],
$$

in a medium with a single fiber family making an angle $\gamma$ with the $X_{1}$-direction, the displacement $G\left(X_{1}, t\right)$ satisfies

$$
G_{t t}=\left(\alpha+\beta \cos ^{2} \gamma\left(3 \cos ^{2} \gamma\left(G_{x}\right)^{2}+6 \sin \gamma \cos \gamma G_{x}+2 \sin ^{2} \gamma\right)\right) G_{x x}
$$

(here for simplicity of notation, we have renamed $X_{1}$ by $x$ in $G\left(X_{1}, t\right) \equiv G(x, t)$ ). The model involves three arbitrary elements, the material parameters $\alpha=2(a+b)>0, \beta=4 q>0$, and the material fiber angle $\gamma \in[0, \pi / 2]$.

When $\gamma= \pm \pi / 2$, i.e., the fibers are aligned along the material $z$-direction, and the PDEs (5.4) reduce to the classical linear wave equations $G_{t t}=\alpha G_{x x}$, in particular, fiber effects vanish. When the fiber angle is $\gamma=0$, i.e., when the wave propagates along the fibers, the equation (5.4) assumes a particularly simple form

$$
G_{t}=\left(\alpha+3 \beta G_{x}^{2}\right) G_{x x} .
$$

We now use the algorithm of Section 2.2 to compute equivalence transformations of the PDEs (5.4). For the sake of simplicity, we denote

$$
k=\tan \gamma \in \mathbb{R}
$$

The PDE family (5.4) thus involves three arbitrary elements: $\alpha, \beta, k$. The corresponding infinitesimal generators of point equivalence transformations (2.21) take the form

$$
\boldsymbol{v}=\xi \frac{\partial}{\partial x}+\tau \frac{\partial}{\partial t}+\eta \frac{\partial}{\partial G}+\zeta^{1} \frac{\partial}{\partial \alpha}+\zeta^{2} \frac{\partial}{\partial \beta}+\zeta^{3} \frac{\partial}{\partial k} .
$$

For the current application, it is sufficient to consider regular equivalence transformations (2.14), so the dependencies of the infinitesimal components are chosen as follows:

$$
\begin{aligned}
\xi & =\xi(x, t, G), \quad \tau=\tau(x, t, G), \quad \eta=\eta(x, t, G), \\
\zeta^{v} & =\zeta^{v}(\alpha, \beta, k), \quad v=1,2,3 .
\end{aligned}
$$

After the solution of determining equations, one obtains eight linearly independent basis generators of equivalence transformations, given by

$$
\begin{gathered}
\boldsymbol{v}_{1}=\frac{\partial}{\partial G}, \quad \boldsymbol{\nu}_{2}=\frac{\partial}{\partial x}, \quad \boldsymbol{v}_{3}=\frac{\partial}{\partial t}, \quad \boldsymbol{v}_{4}=t \frac{\partial}{\partial G}, \\
\boldsymbol{v}_{5}=t \frac{\partial}{\partial t}+x \frac{\partial}{\partial x}+G \frac{\partial}{\partial G}, \quad \boldsymbol{v}_{6}=-\frac{1}{2} t \frac{\partial}{\partial t}+\alpha \frac{\partial}{\partial \alpha}+\beta \frac{\partial}{\partial \beta}, \\
\boldsymbol{v}_{7}=-x \frac{\partial}{\partial x}-2 \alpha \frac{\partial}{\partial a}-\frac{4 \beta}{k^{2}+1} \frac{\partial}{\partial \beta}+k \frac{\partial}{\partial k} \\
\boldsymbol{v}_{8}=-x \frac{\partial}{\partial G}+\frac{2 \beta k}{\left(k^{2}+1\right)^{2}} \frac{\partial}{\partial \alpha}+\frac{4 \beta k}{k^{2}+1} \frac{\partial}{\partial \beta}+\frac{\partial}{\partial k} .
\end{gathered}
$$

The transformation generator $\boldsymbol{\nu}_{8}$ in (5.7) corresponds to shifts in $k \equiv \tan \gamma$. The global group (2.26) is found by integration, and has the form

$$
\begin{array}{ll}
\bar{x}=x, \quad \bar{t}=t, \quad \bar{G}=G-S x, & \tan \bar{\gamma}=\tan \gamma+\mu, \\
\bar{\alpha}=\alpha+2 \beta \cos ^{4} \gamma\left(\frac{s^{2}}{2}+\mu \tan \gamma\right), & \bar{\beta}=\beta \cos ^{4} \gamma\left(\tan ^{2} \gamma+2 \mu \tan \gamma+\mu^{2}+1\right)^{2},
\end{array}
$$


with a group parameter $\mu \in \mathbb{R}$. Choosing $\mu=-k=-\tan \gamma$, one invertibly maps the $\operatorname{PDE}$ (5.4) into the form (5.5) with $\bar{\gamma}=0$. This equivalence transformation is given by

$$
x=\bar{x}, \quad t=\bar{t}, \quad G=\bar{G}-x \tan \gamma, \quad \alpha=\bar{\alpha}+\bar{\beta} \tan ^{2} \gamma, \quad \beta=\bar{\beta} \cos ^{-4} \gamma .
$$

The substitution of (5.8) into the full PDE (5.4) yields

$$
\bar{G}_{\bar{t} \bar{t}}=\left(\bar{\alpha}+3 \bar{\beta}\left(\bar{G}_{\bar{x}}\right)^{2}\right) \bar{G}_{\bar{x} \bar{x}}
$$

with

$$
\bar{\alpha}=\alpha+\frac{\beta}{4} \sin ^{2} 2 \gamma, \quad \bar{\beta}=\beta \cos ^{4} \gamma,
$$

which is the "simpler" equation (5.5).

Note that the equation (5.9) has the parameter $\bar{\alpha}^{*}$ that may not be positive. In particular, loss of hyperbolicity may occur when the coefficient of $G_{x x}$ becomes negative [62]. A sufficient condition for the loss of hyperbolicity not to happen under the indicated transformation is given by

$$
\sin ^{2}(2 \gamma)<\frac{4 \alpha}{\beta} \text {. }
$$

The condition (5.10) is satisfied for all fiber orientations $\gamma$, for example, in the common situation of relatively weak fiber contributions, $\beta<4 \alpha$. In this case, obvious nondimensionalizing scaling transformations

$$
\widehat{x}=\frac{\bar{x}}{L}, \quad \widehat{t}=\frac{\sqrt{\bar{\alpha}} \bar{t}}{L}, \quad \widehat{G}=\sqrt{\frac{3 \bar{\beta}}{\bar{\alpha}}} \frac{\bar{G}}{L}, \quad L \in \mathbb{R}^{+}
$$

of the PDE family (5.9) can be used to further map all these equations into a single nonlinear wave equation

$$
\widehat{G}_{\hat{t} \hat{t}}=\left(1+\left(\widehat{G}_{\widehat{x}}\right)^{2}\right) \widehat{G}_{\widehat{x} \widehat{x}} .
$$

Thus through systematically computed equivalence transformations, the PDE family (5.4) with three constitutive parameters is invertibly reduced to a wave equation (5.12) with no arbitrary elements. For properties of the PDE (5.12), sample numerical solutions of boundary value problems, and the extended versions of the model, see [62].

\section{Discussion and concluding remarks}

Lie symmetries and related ideas provide a set of powerful tools in the realm of mechanics of materials. While the Lie theory and related methods and ideas applicable to ODE/PDE models have been significantly extended to include nonlocal and approximate symmetries, methods of moving frames and co-frames, and various approaches to produce invariant (in some sense) numerical methods (e.g., [5,6,64-70], it us usually the most basic and fundamental ideas and simplest Lie symmetry-based computations that yield most useful results for complex PDE models in nonlinear mechanics. In particular, symmetry ideas prove useful in many different ways to construct a constitutive law of a given material from experimental results, in terms of a functional relation between control variables and additional parameters. Thereby, symmetries are used to solve an inverse problem, assuming the measured data have a structure akin to a Lie group, when considering a continuous range of variation of the adopted parameters. 
Once a set of constitutive equations (in other words, constitutive parameters and/or functions) is determined, equivalence transformations can be systematically sought and applied to relate and simplify the forms of constitutive dependencies, thereby leading to model reduction. Lie groups of symmetries, equivalence transformations, and related structures can be computed algorithmically using symbolic softwares, such as the GeM package for Maple [16-19] and other packages.

The knowledge of the Lie symmetries of the constructed constitutive model allows as a next step to extrapolate the material response for a continuous variation of the inherent parameters. This powerful and systematic methodology is especially interesting to predict the material's response for values of the parameters that are not easily accessible in experiments. In the context of model reduction, Lie symmetries constitutive a powerful tool revealing the intrinsic invariance properties of boundary value problems formulated in the framework of TIP (thermodynamics of irreversible processes), and using these invariance properties to exhibit relations between the constitutive model variables and parameters having a physical meaning reflected by the free energy density and the dissipation potential. The present methodology exploiting Lie symmetries in a combined direct and inverse manner can potentially be applied to a wide class of materials with a complex rheology, including polymers, metals, ceramics, metallic and polymeric foams and constitutive laws, such as nonlinear elasticity, viscoelasticity and viscoplasticity, with or without damage.

For the above-discussed applications, work on the applications of symmetry-based methods will continue in various directions:

- Development of constitutive models synthesizing data issued from measurements, thereby using symmetry methods as an interpolation method, allowing to rely on a minimum amount of data.

- Applications in material modelling, since a natural framework for building constitutive models (including multiphysical couplings, material nonlinearities) is the thermodynamics of irreversible processes relying on energetic and dissipative potentials, the proposed Lagrangian formulation of dissipative constitutive laws proves convenient to articulate symmetries, conservation laws (invariance relations) and master responses giving a graphical representation of those invariance relations amongst variables and parameters.

- Extrapolation of existing models to enable predictions of the variation of parameters in a range where measurements can not easily be done.

- Reduction of the number of parameters in models to the minimum using equivalence transformations.

- Development of specialized symmetry-preserving numerical schemes for complex boundary value problems in continuum mechanics and thermodynamics.

$A_{i}$ : Lie algebra $\left\{\boldsymbol{v}_{i}^{1}, \ldots, \boldsymbol{v}_{i}^{n_{i}}\right\}$ of the equation $\Delta_{i}=0 ; \boldsymbol{\Delta}=0$ : set of constitutive equations $\left\{\Delta_{1}=0, \cdots, \Delta_{n}=0\right\} ; \varepsilon$ : strain; $\dot{\varepsilon}$ : strain rate; $G_{i}^{o b s}: i^{\text {th }}$ Lie group formulated from experimental data; $\Phi_{i}^{p_{k}}$ : transformation function of the parameter $p_{k}$ for the $i^{\text {th }}$ experimental group; $\Phi_{i}^{u_{k}}$ : transformation function of the observable variable $u_{k}$ for the $i^{\text {th }}$ experimental group; $K_{i}$ : set of indexes $k$ such that $\boldsymbol{v}_{k}^{o b s}$ is a symmetry of $\Delta_{i}=0 ; \log x$ : natural logarithm of $x ; m$ : number of parameters (range of $\boldsymbol{p}) ; m_{i}$ : range of $K_{i} ; \mu$ : parameter of a Lie group; $n$ : number of observables (range of $\boldsymbol{u}$ ); $n_{i}$ : number of generators in the Lie algebra $A_{i} ; \phi^{x}: x$-component of a symmetry generator; $\boldsymbol{p}$ : set of parameters $\left\{p_{1}, \cdots, p_{m}\right\} ; P_{j}$ : domain of $p_{j}$ (interval of variation); $\mathcal{P}$ : Cartesian product $P_{1} \times P_{2} \cdots \times P_{m}$, domain of $\boldsymbol{p} ; \mathrm{pr}^{()} \boldsymbol{v}$ : prolongation of the vector field $\boldsymbol{v}$ (no order specified); $\operatorname{pr}^{(\kappa)} \boldsymbol{v}: \kappa$-order prolongation of the vector field $\boldsymbol{v} ; Q$ : activation energy; $q$ : number of "observed" symmetries; $R$ : perfect gas constant; $\sigma$ : Cauchy stress; $t$ : time; $t_{R}$ : time at rupture; $T$ : absolute temperature; $\boldsymbol{u}$ : set of observables
} 
$\left\{u_{1}, \cdots, u_{n}\right\} ; \boldsymbol{u}^{-j}$ : set of observables $\left\{u_{1}, \cdots, u_{j-1}, u_{j+1}, \cdots, u_{n}\right\} ; U_{i}$ : domain of $u_{i} ; \mathcal{U}$ : Cartesian product $U_{1} \times U_{2} \cdots \times U_{n}$, domain of $\boldsymbol{u} ; \mathcal{U}^{-j}$ : Cartesian product $U_{1} \times \cdots U_{j-1} \times U_{j+1} \cdots \times U_{n}$, domain of $\boldsymbol{u}^{-j} ; \boldsymbol{v}_{i}^{\text {obs }}$ : generator of the Lie group $G_{i}^{o b s} ; v_{i}^{j}: j^{\text {th }}$ generator of the Lie algebra $A_{i} ; \bar{x}$ : transformed value of the quantity $x$ by a Lie group.

\section{Acknowledgements}

The authors thank Vincent Magnenet for his contribution to parts of the theoretical developments and results presented in this paper.

\section{Authors' contributions}

Authors' contributions: all authors contributed approximately equally to 964 this manuscript.

\section{Funding}

A. C. is grateful to NSERC of Canada for research support through a Discovery grant RGPIN-2019-05570. Other funding: no funding.

\section{Availability of data and material}

Availability of data and materials: data used in graphs and tables is available in previous publications: see Refs. [41,54,61].

\section{Competing interests}

Not applicable

\section{Author details}

'LEM3, UMR CNRS 7239, Université de Lorraine, 7, rue Félix Savart, 57073 Metz, France, ${ }^{2}$ Department of Mathematics and Statistics, University of Saskatchewan, Room 227 McLean Hall, 106 Wiggins Road, Saskatoon, SK S7N 5E6, Canada.

Received: 18 December 2019 Accepted: 29 November 2020

Published online: 05 January 2021

\section{References}

1. Auffray N, Le Quang H, He QC. Matrix representations for 3D strain-gradient elasticity. Journal of the Mechanics and Physics of Solids. 2013;61(5):1202-23.

2. Yong-Zhong H, Del Piero G. On the completeness of the crystallographic symmetries in the description of the symmetries of the elastic tensor. Journal of Elasticity. 1991;25(3):203-46.

3. Zheng QS, Boehler JP. The description, classification, and reality of material and physical symmetries. Acta Mechanica. 1994;102(1-4):73-89.

4. Hamel G. Die Lagrange-Eulerschen Gleichungen der Mechanik. Z. Mathematik und Physik. 1904;50:1-57.

5. Olver PJ. Applications of Lie Groups to Differential Equations, vol. 107. : Springer; 2000.

6. Bluman GW, Cheviakov AF, Anco SC. Applications of symmetry methods to partial differential equations, vol. 168.: Springer; 2010.

7. Noether E. Invariante Variationsprobleme, Nachr. Konig. Gesell. Wissen. Gottingen, Math.-Phys. Kl., 1918.

8. Sciarra G, Dell-Isola F, laniro N, Madeo A. A variational deduction of second gradient poroelasticity part I: General theory. Journal of Mechanics of Materials and Structures. 2008;3(3):507-26.

9. Weyl H. The Classical Groups: their Invariants and Representations, vol. 45. : Princeton University Press; 1946

10. Fulton W, Harris J. Representation Theory: a First Course. : Springer Verlag; 1991.

11. Magnenet V, Rahouadj R, Ganghoffer JF. A new methodology for determining the mechanical behavior of polymers exploiting Lie symmetries: application to a stick-like material. Mechanics of Materials. 2009;41 (9):1017-24.

12. Barenblatt Gl. Scaling, Self-similarity and Intermediate Asymptotics. : Cambridge University Press; 1996.

13. Barenblatt GI. Scaling phenomena in fatigue and fracture. International Journal of Fracture. 2006;138:19-35

14. Carpinteri A, Paggi M. A unified interpretation of the power laws in fatigue and the analytical correlations between cyclic properties of engineering materials. International Journal of Fatigue. 2009;31:1524-31.

15. Lisle I. Equivalence transformations for classes of differential equations, Ph.D. thesis, University of British Columbia 1992.

16. Cheviakov AF. Symbolic computation of equivalence transformations and parameter reduction for nonlinear physical models, Computer Physics Communications 2017:220 (Supplement C) :56 - 73. https://doi.org/10.1016/..cpc.2017. 06.013. http://www.sciencedirect.com/science/article/pii/S0010465517301959.

17. Cheviakov AF. GeM software package for computation of symmetries and conservation laws of differential equations. Computer Physics Communications. 2007;176(1):48-61.

18. Cheviakov AF. Computation of fluxes of conservation laws. Journal of Engineering Mathematics. 2010;66(1-3):153-73.

19. Cheviakov AF. Symbolic computation of local symmetries of nonlinear and linear partial and ordinary differential equations. Mathematics in Computer Science. 2010;4(2-3):203-22.

20. Müller I. A thermodynamic theory of mixtures of fluids. Archive for Rational Mechanics and Analysis. 1968;28(1):1-39.

21. Müller I. On the entropy inequality. Archive for Rational Mechanics and Analysis. 1967;26(2):118-41.

22. Müller I. A new systematic approach to non-equilibrium thermodynamics. Pure and Applied Chemistry. 1970;22(34):335-42.

23. Müller I. The coldness, a universal function in thermoelastic bodies. Archive for Rational Mechanics and Analysis. 1971;41(5):319-32.

24. Müller I. Die Kaltefunktion, eine universelle Funktion in der Thermodynamik viskoser warmeleitender Flussigkeiten. Archive for Rational Mechanics and Analysis. 1971;40(1):1-36. 
25. Liu I-S. Method of Lagrange multipliers for exploitation of the entropy principle. Archive for Rational Mechanics and Analysis. 1972;46(2):131-48.

26. Cheviakov AF, Heß J. A symbolic computation framework for constitutive modelling based on entropy principles. Applied Mathematics and Computation. 2018;324:105-18.

27. Heß J, Cheviakov AF. A solution set-based entropy principle for constitutive modeling in mechanics. Continuum Mechanics and Thermodynamics. 2019:31(3):775-806.

28. Ganghoffer J-F, Magnenet V, Rahouadj R. Relevance of symmetry methods in Mechanics of Materials. International Journal of Engineering Mathematics. 2010;66(1):103-19.

29. Sahin D, Antar N, Ozer T. Lie group analysis of gravity currents. Nonlinear Analysis: Real World Applications. 2010;11(2):978-94.

30. Ozer T. Symmetry group analysis and similarity solutions of variant nonlinear long-wave equations. Chaos, Solitons and Fractals. 2008;38:722-30.

31. Magnenet V, Ganghoffer J-F, Rahouadj R, Cunat C. Continuous symmetries and constitutive laws of dissipative materials within a thermodynamic framework of relaxation. part i: formal aspects, International Journal of Plasticity (23) 2007:87-113.

32. Hydon PE, Hydon PE. Symmetry methods for differential equations: a beginner's guide, vol. 22.: Cambridge University Press; 2000.

33. Ovsiannikov LV. Group Analysis of Differential Equations. : Academic Press; 2014.

34. Meleshko SV. Generalization of the equivalence transformations. Journal of Nonlinear Mathematical Physics. 1996;3(12):170-4.

35. Popovych RO, Ivanova NM, Eshraghi H. Group classification of (1+ 1)-dimensional Schrödinger equations with potentials and power nonlinearities. Journal of Mathematical Physics. 2004;45(8):3049-57.

36. Saint-Antonin F. Techniques de l'ingénieur, Vol. M140, 1995, Ch. Essais de fluage.

37. Kim WG, Kim SH, Ryu WS. Evaluation of Monkman-Grant Parameters for Type $316 \mathrm{LN}$ and modified 9Cr-Mo Stainless Steels. KSME International Journal. 2002;16(11):1420-7.

38. Totemeier TC, Tian H, Simpson JA. Effect of Normalization Temperature on the Creep Strength of Modified 9Cr-1Mo Steel. Metallurgical and Materials Transactions. 2006;37A:1519-25.

39. Kimura K, Kushima H, Sawada K. Long-term creep deformation property of modified 9Cr-1Mo steel. Materials Science and Engineering A. 2009;510-511:58-63.

40. Skrzypek JJ, Hetnarski RB. Plasticity and Creep.: CRC Press; 1993.

41. Ganghoffer J-F, Magnenet V, Rahouadj R. Relevance of symmetry methods in mechanics of materials. Journal of Engineering Mathematics. 2010;66(1-3):103-19.

42. Maugin GA. The Thermodynamics of Nonlinear Irreversible Behaviours. An Introduction. : World Scientific; 1999.

43. Callen HB. Thermodynamics and an Introduction to Thermostatistics. 2nd ed. : John Wiley \& Sons; 1985.

44. Cunat C. The DNLR approach and relaxation phenomena. Part 1: Historical account and DNLR formalism. Mechanics of time-dependant materials. 2001;5(1):39-65.

45. Magnenet V, Rahouadj R, Ganghoffer JF, Cunat C. Continuous symmetries and constitutive laws of thermoelasto(visco) plastic materials within a thermodynamic framework of relaxation. Part l: Formal aspects, Internal Journal of Plasticity (23) 2007; 87-113.

46. Rahouadj R, Ganghoffer JF, Cunat C. A thermodynamic approach with internal variables using Lagrange formalism. Part I: General framework, Mechanics Research Communications. 2003;30(2):109-17.

47. Rahouadj R, Ganghoffer JF, Cunat C. A thermodynamic approach with internal variables using Lagrange formalism. Part II: Continuous symmetries in the case of the time-temperature equivalence, Mechanics Research Communications. 2003;30(2):119-23.

48. Magnenet V, Rahouadj R, Ganghoffer JF, Cunat C. Master curves for viscous media predicted from Lie symmetry analysis, in: Proc. of the Symposium on Trends in Applications of Mathematics to Mechanics, Darmstadt, Germany, 2004.

49. Biot MA. Variational and Lagrangian Methods in Viscoelasticity. : Springer-Verlag; 1956.

50. Biot MA. Variational principles in irreversible thermodynamics with application to viscoelasticity, Physical Review 97 (6).

51. ZH, Some extremum principles in irreversible thermodynamics with applications to continuum mechanics, in: R. Sneddon, I.N. Hill (Ed.), Progress in Solid Mechanics, Vol. IV., 1963.

52. Germain P. Functional concepts in continuum mechanics. Meccanica. 1998;33(5):433-44.

53. Magnenet V, Rahouadj R, Ganghoffer JF. A new methodology for determining the mechanical behavior of polymers exploiting Lie symmetries: application to a stick-like material, Mechanics of Materials (To be published).

54. Magnenet V, Rahouadj R, Ganghoffer JF, Cunat C. Continuous symmetry analysis of a dissipative constitutive law: application to the time-temperature superposition, European Journal of Mechanics A/Solids (28) 2009; 744-751.

55. Hotta A, Terentjev EM. Long-time stress relaxation in polyacrylate nematic liquid crystalline elastomers, Journal of Physics: Condensed Matter 13.

56. C. Laot, Gas transport properties in polycarbonate, Ph.D. thesis, Virginia Polytechnic Institute (2001).

57. Ricco T, Pegoretti A. Nonlinear dynamic behavior of rubber compounds: construction of dynamic moduli generalized master curves, Polymer Engineering and Science 40.

58. Meyer A, Busch R, Schober H. Time-temperature superposition of structural relaxation in a viscous metallic liquid, Physical Review Letters 83.

59. O'Connell PA, McKenna GB. Large deformation response of polycarbonate: time temperature, time aging time and time strain superposition, Polymer Engineering and Science 37.

60. Nicholson LM, Whistley KS, Gates TS. Crosslink density and molecular weight effects on the viscoelastic response of a glassy high-performance polyimide, Polymer Preprints 42

61. Magnenet V, Rahouadj R, Ganghoffer J-F. Symmetry analysis and invariance relations in creep. Mathematics and Mechanics of Solids. 2014;19(8):988-1010. 
62. Cheviakov A, Ganghoffer J-F. One-dimensional nonlinear elastodynamic models and their local conservation laws with applications to biological membranes, Journal of the Mechanical Behavior of Biomedical Materials.

63. Cheviakov A, Ganghoffer J-F, Jean SS. Fully non-linear wave models in fiber-reinforced anisotropic incompressible hyperelastic solids. International Journal of Non-Linear Mechanics. 2015;71:8-21.

64. Cartan E. La methode du repere mobile, la theorie des groupes continus et les espaces generalizes, exposes de geometrie, vol. v, V, Hermann, Paris.

65. Fels M, Olver PJ. Moving coframes: I. a practical algorithm. Acta Applicandae Mathematica. 1998;51(2):161-213.

66. Fels M, Olver PJ. Moving coframes: li. regularization and theoretical foundations. Acta Applicandae Mathematica. 1999;55(2):127-208.

67. Razafindralandy D, Hamdouni A, AlSayed N. Lie-symmetry group and modeling in non-isothermal fluid mechanics. Physica A: Statistical Mechanics and its Applications. 2012;391(20):4624-36.

68. Ibragimov NH. CRC handbook of Lie group analysis of differential equations, vol. 3. : CRC Press; 1995.

69. Cheviakov A, Dorodnitsyn V, Kaptsov E. Invariant conservation law-preserving discretizations of linear and nonlinear wave equations. Journal of Mathematical Physics. 2020;61(8):081504.

70. Chhay M, Hoarau E, Hamdouni A, Sagaut P. Comparison of some lie-symmetry-based integrators. Journal of Computational Physics. 2011;230(5):2174-88.

71. Soderberg CR. The interpretation of creep tests for machine design, Trans. ASME 58.

72. Rabotnov JN. Creep analysis of structural members (in Russian), Izv. Acad. Nauk SSSR, Otd. Tekhn. Nauk 6.

73. Davenport CC. Correlation of creep and relaxation properties of copper, Journal of Applied Mechanics 5 (2).

74. Rabotnov JN. Creep Problems in Structural Members. : Nauka; 1966 (in Russian).

75. Penny RK, Marriott DL. Design for Creep. : McGraw-Hill; 1971.

76. Boyle JT, Spence J. Stress Analysis of Creep. : Butterworths; 1983.

77. Ludwik P. Elemente der Technologischen Mechanik. : Springer; 1909.

78. Nadai A. The influence of time upon creep, the hyperbolic sine creep law. Stephen Timoshenko Anniversary. 1938;15570 .

\section{Publisher's Note}

Springer Nature remains neutral with regard to jurisdictional claims in published maps and institutional affiliations.

\section{Submit your manuscript to a SpringerOpen ${ }^{\circ}$ journal and benefit from:}

- Convenient online submission

- Rigorous peer review

Open access: articles freely available online

- High visibility within the field

- Retaining the copyright to your article

Submit your next manuscript at $>$ springeropen.com 